\title{
Kinky Perceived Demand Curves and keynes-Negishi Equilibria
}

Citation for published version (APA):

Drèze, J., \& Herings, P. J. J. (2008). Kinky Perceived Demand Curves and keynes-Negishi Equilibria. International Journal of Economic Theory, 4, 207-246. https://doi.org/10.1111/j.1742-7363.2008.00072.x

Document status and date:

Published: 01/01/2008

DOI:

10.1111/j.1742-7363.2008.00072.x

Document Version:

Publisher's PDF, also known as Version of record

Document license:

Taverne

Please check the document version of this publication:

- A submitted manuscript is the version of the article upon submission and before peer-review. There can be important differences between the submitted version and the official published version of record.

People interested in the research are advised to contact the author for the final version of the publication, or visit the DOI to the publisher's website.

- The final author version and the galley proof are versions of the publication after peer review.

- The final published version features the final layout of the paper including the volume, issue and page numbers.

Link to publication

\footnotetext{
General rights rights.

- You may freely distribute the URL identifying the publication in the public portal. please follow below link for the End User Agreement:

www.umlib.nl/taverne-license

Take down policy

If you believe that this document breaches copyright please contact us at:

repository@maastrichtuniversity.nl

providing details and we will investigate your claim.
}

Copyright and moral rights for the publications made accessible in the public portal are retained by the authors and/or other copyright owners and it is a condition of accessing publications that users recognise and abide by the legal requirements associated with these

- Users may download and print one copy of any publication from the public portal for the purpose of private study or research.

- You may not further distribute the material or use it for any profit-making activity or commercial gain

If the publication is distributed under the terms of Article $25 \mathrm{fa}$ of the Dutch Copyright Act, indicated by the "Taverne" license above, 


\title{
Kinky perceived demand curves and Keynes-Negishi equilibria
}

\author{
Jacques H. Drèze* and P. Jean-Jacques Herings ${ }^{\dagger}$
}

\begin{abstract}
The label "Keynes-Negishi equilibria" is attached here to equilibria in a monetary economy with imperfectly competitive product and labor markets where business firms and labor unions hold demand perceptions with kinks: as posited in Negishi's 1979 book Microeconomic Foundations of Keynesian Macroeconomics. Such equilibria are defined in a general equilibrium model, and shown to exist. Methodological implications are briefly discussed in a concluding section.
\end{abstract}

Key words equilibrium, imperfect competition, perceived demands, kinky demand, pricing rules, union wage model, union objectives, cash-in-advance

JEL classification D50, E12, J51

Accepted 16 October 2007

In economic science general equilibrium theory plays a role similar to that played in university curricula by mathematics and language studies. It must certainly have its own discipline (i.e. the demonstration of the consistency of its theoretical structure), but at the same time it should also offer services to other branches of study. To tell the story of general equilibrium theory, therefore, one must talk of its applications as well as of its pure theory.

Takashi Negishi (1972, p. vii)

\section{Introduction}

\subsection{Background}

In two papers and a book, Takashi Negishi $(1974,1976,1979)$ has developed "microfoundations for Keynesian macroeconomics”. He writes (1979, p. 2): “The Keynesian economic system is a fixprice system in the sense of Hicks (1974) that prices are independent

\footnotetext{
*Center of Operation Research and Econometrics Université Catholique de Louvain, Louvain-la-Neuve, Belgium. Email: dreze@core.ucl.ac.be

${ }^{\dagger}$ Department of Economics, Maastricht University, Maastricht, the Netherlands.

The authors would like to thank an anonymous referee for helpful comments and suggestions. P.J.-J. Herings would like to thank the Netherlands Organization for Scientific Research for financial support.
} 
of demand and supply. One should not be satisfied, however, merely by this statement. One has to explain why prices (and wages) are independent of demand and supply."

Negishi's own explanation rests on kinks in the demand functions perceived by the agents. The concept of "perceived demand functions" is introduced in Negishi (1961) 1 and is developed further in Negishi (1972). It provides a simpler and more realistic foundation for general equilibrium modeling of monopolistic or imperfect competition than objective demand functions. ${ }^{2}$ It is applicable both to the situation of a firm concerned with assessing the implications of alternative prices for output demand; and to the situation of a union concerned with assessing the implications of alternative wages for labor demand.

In both cases, the perceived demand curves summarize the information available to the agent. Typically, however, that information may be limited, implying uncertainty about market reactions; and it may suggest different market reactions for price decreases versus price increases. This last feature is conducive to a kink in the perceived demand curve at the current output point. It was justified in Sweezy (1939) by asymmetrical reactions of competitors, in Stiglitz (1984) by asymmetrical search behavior of consumers. Drèze (1979) notes that uncertainty about demand elasticity leads risk-averse firms to behave as if they face a kinky demand curve.

It was Negishi's original "perception" that kinky perceived demand curves could explain the price-wage stickiness associated with Keynesian equilibria. ${ }^{3}$ That idea is treated most fully in his 1979 book, which includes a number of interesting side-developments. The hurried reader will find the gist of the argument in chapter 7, entitled "Kinked demand curves and Keynesian equilibrium" (pp. 87-97).

That chapter analyzes a simplified economy with two "industries" supplying, respectively, one consumer good and one investment good. Each industry is modeled through a representative firm, with a short-run neoclassical production function relating output to uni-dimensional labor input. A fixed share of profits is saved, and savings should match an exogenous level of real investment demand. Labor is supplied by identical consumers/workers. Both the representative firms and the representative worker are endowed with asymmetrical perceived price elasticities; these are assumed equal to zero in the case of a decrease in goods or labor supply, or equal to a finite strictly negative constant in the case of an increase. Assuming that the price elasticities relevant to supply increases are greater than unity in absolute value, Negishi proves the existence of a Keynesian equilibrium, ${ }^{4}$ and concludes with the unexpected corollary that "real wages can be lower at an underemployment equilibrium than at the full employment equilibrium" (Negishi 1979, p. 97).

\subsection{Outline and summary}

The purpose of this paper is to prove (27 years later!) the existence of a Keynes-Negishi equilibrium for a model and under assumptions of the kind used in modern general equilibrium

\footnotetext{
${ }^{1}$ At that time, Negishi was still a graduate student!

${ }^{2}$ See Negishi (1972, p. 107) or Dehez et al. (2003, p. 220).

${ }^{3}$ More recently, that theme has been revived by Woodford (1991). Note, however, that Woodford (2003) ignores it altogether.

${ }^{4}$ The equilibrium is such that exogenous shocks to aggregate demand (to investment, in Negishi's model) result in quantity adjustments at unchanged prices.
} 
theory. This purpose is clearly in the spirit of Negishi $(1961,1972)$. It calls for multiple goods or types of labor, and for heterogeneous agents. Negishi's program adds two requirements: monetary exchange and organized labor.

The treatment of these two topics is not found in chapter 7 of Negishi (1979); instead, these topics receive specific attention in separate chapters. ${ }^{5}$ We return in Section 7 below to the methodological premises of these chapters. Suffice it to record here that: (i) we adhere to the "rule of the game" (Negishi 1979, p. 28) that "all exchanges are assumed to be monetary in the sense of Clower (1967)"; that is, money is demanded for transaction purposes, as exemplified by the "cash-in-advance" model; (ii) we concur that "a trade union is not a monopolistic firm but an association of heterogeneous workers" (Negishi 1979, p. 7). Our own treatment of these two topics (in Sections 4 and 5) aims again at generality.

This paper rests on the concept of "pricing rules" introduced in the seminal paper by Dierker et al. (1985). A pricing rule is a correspondence associating with every technologically efficient production plan for a firm the set of price vectors at which the firm would supply that production. An application of that concept to "imperfect competition à la Negishi” is developed in Dehez et al. (2003), starting from the pioneering formulation in Negishi (1961, 1972). The pricing rule in Dehez et al., reviewed in Section 2 below, is derived from the conditions for profit maximization under a perceived demand function congruent with given market signals (an allocation and a price vector). Here, we derive (in Section 3) the pricing rule from compatibility with profit maximization at a kink of a perceived demand function, thus allowing demand elasticities to differ as between price hikes and price cuts. We derive the set of inequalities that is necessary and sufficient for neither price increases nor price cuts to entail higher profits, under a technologically efficient production plan, given an allocation and market prices. From these, we derive simpler inequalities that lead to sufficient conditions. Our pricing rule is the correspondence defined by the latter inequalities. The formulation is quite general and applicable to perfectly as well as imperfectly competitive firms, whose demand perceptions may or may not display kinks. We introduce kinky perceived demand functions in Section 3, after introducing the model in Section 2.

Section 4 brings in unions; that is, "associations of heterogeneous workers". The primitives of the economy are enlarged with a set of unions, each defined by its membership and by the set of labor markets on which it sets wages. Each union is endowed with labor demand perceptions of the markets where it sets wages, and these perceptions typically entail kinks.

The objective of the union has been the subject of a vast literature, surveyed in Negishi (1979), Oswald (1985) and Farber (1986). Our formulation, aiming at generality with consistency, assumes that unions act in the interest of their heterogeneous members. More precisely, we assume that wages set by a union are Pareto efficient from the viewpoint of the given set of members. Interestingly, this guiding principle is analogous to that advocated in some literature on decision criteria for business firms operating in an incomplete markets framework. Starting with Drèze (1974), ${ }^{6}$ it has been recognized that a firm aiming at Pareto

\footnotetext{
${ }^{5}$ Cf. Chapter 10, "Vulnerability to inflation"; and Chapter 17, "Employment, wages and trade unions".

${ }^{6}$ See, for example, Magill and Quinzii (1996).
} 
efficiency from the viewpoint of the shareholders should evaluate profits (not fully priced on the incomplete markets) at shadow prices defined as weighted averages of the corresponding shadow prices of shareholders, with weights given by shareholdings. A comparable property holds for wage-setting unions, which should attach to their wage-unemployment trade-off a shadow price (reservation wage) averaging the reservation wages of the union's members.

It is shown in Section 4 that unions are led by this criterion to adopt pricing rules formally analogous to those of imperfectly competitive firms. The substantive difference lies in the objectives (Pareto efficiency versus profit maximization). ${ }^{7}$

Section 5 brings in money. The simplest way to do so, which we borrow from Drèze and Polemarchakis $(1999,2001 \mathrm{a}, \mathrm{b})$, is to introduce non-interest bearing "inside" money, ${ }^{8}$ produced at no cost by a (central) bank. The bank prints money as demanded by the agents (households and firms), and lends it against promises to reimburse with interest. "Inside" money means that the bank keeps balanced accounts: the quantity of money outstanding is equal to the sum of claims on the agents held by the bank. The bank sets nominal interest rates: this is the "pricing rule" of the bank, here treated as a primitive. The interest collected by the bank corresponds to bank profits, which are distributed to agents as dividends. "Cash-in-advance" means that the agents (households and firms) face different net prices for purchases (paid for at the time of transaction) and for sales (whose proceeds accrue at the end of the period). There thus exist kinks in the budget or profit equations, also making the presence of kinks ubiquitous in the present paper.

Sections 3-5 thus cover with much generality the existence part of Negishi's program, culminating in the general result of Section 6. Some applications and methodological issues are briefly discussed in the concluding Section 7.

\subsection{Technical guideline}

This paper grew out of the realization (in July 2006!) that scattered results on general equilibrium with imperfect competition à la Negishi, with firms implementing Pareto efficiency for their shareholders, or with inside money could be: (i) extended to kinky demand perceptions; and (ii) combined in an integrated framework. ${ }^{9}$ Both achievements are of independent interest in their own right. Not surprisingly, a host of technical hurdles sprang up in the course of implementing that program. To guide readers through these hurdles, we start each of the innovative Sections 3, 4 and 5 with an elementary example (already introduced in Section 2.4) that illustrates the extensions to the pricing rules. The technical developments themselves are then presented in lemmas, proved in the Appendix.

Because the paper by Dehez et al. (2003) provided our starting point, we were led to rely on the technical tool of pricing rules: inescapable there to deal with non-convexities in production. Each of the three innovative sections, Sections 3, 4 and 5, is devoted essentially

\footnotetext{
${ }^{7}$ Under incomplete markets, the difference in objectives disappears if firms adopt the Drèze criterion.

${ }^{8}$ This specification is thus sharply different from the models of "outside" money, discussed for instance in Negishi (1972).

9 The realization was triggered by our own involvement in the earlier work in the three areas at stake, in particular Dehez et al. (2003) for Section 3, Drèze (1974) for Section 4, and Drèze and Polemarchakis (2001b) for Section 5. This also resulted in an excessive inclination towards self-citations, for which we solicit the readers' indulgence.
} 
to develop pricing rules (of firms and/or unions) for the extended frameworks. Readers are reminded (repeatedly!) that pricing rules themselves are a tool of the theorist, not a behavioral characterization of the agents.

\section{An elementary real model}

\subsection{Primitives}

We shall throughout deal with a standard abstract economy, whose real side consists of: ${ }^{10}$

(a) A commodity space $\mathbb{R}^{L}$, with coordinates (commodities) indexed by $l \in \mathcal{L} .^{11}$

(b) A set of $J$ firms indexed by $j \in \mathcal{J}$, each defined by a production set $Y^{j} \subset \mathbb{R}^{L}$ (closed, convex) satisfying $Y^{j}+\mathbb{R}_{-}^{L} \subset Y^{j}$ (free disposal) and $Y^{j} \cap \mathbb{R}_{+}^{L}=\{0\}$ (absence of free production, possibility of inaction).

(c) A set of $H$ households indexed by $h \in \mathcal{H}$; each household is defined by the tuple ( $X^{h}$, $\succeq_{h}, e^{h}, \theta^{h}$ ). The tuple consists of a consumption set $X^{h} \subset \mathbb{R}^{L}$ (closed, convex and bounded from below), a preference preordening $\succeq_{h}$ on $X^{h}$ (complete, continuous, convex and weakly monotone), a vector of initial endowments $e^{h}$ for which there exists $\underline{x}^{h} \in X^{h}$ such that $\underline{x}^{h} \ll e^{h}$, and a vector of ownership fractions $\theta^{h}=\left(\theta^{h j}\right)_{j \in \mathcal{J}}$ in the $J$ firms, $0 \leq \theta^{h j} \leq 1$, where for each $j, \sum_{h} \theta^{h j}=1 .^{12}$

A consumption plan for household $h$ is $x^{h} \in X^{h}$. A production plan for firm $j$ is $y^{j} \in Y^{j}$. An allocation $a$ is a tuple

$$
(x, y)=\left(\left(x^{h}\right)_{h \in \mathcal{H}},\left(y^{j}\right)_{j \in \mathcal{J}}\right) \in \prod_{h \in \mathcal{H}} X^{h} \times \prod_{j \in \mathcal{J}} Y^{j} .
$$

The allocation $a$ is feasible if it satisfies

$$
\sum_{h \in \mathcal{H}} x^{h} \leq \sum_{h \in \mathcal{H}} e^{h}+\sum_{j \in \mathcal{J}} y^{j} .
$$

The set $A$ denotes the set of feasible allocations. The aggregate production set is $Y=\sum_{j \in \mathcal{J}} Y^{j}$. We further assume the following.

(A.1) The production process is irreversible: $Y \cap-Y=\{0\}$.

This assumption implies that the set of feasible allocations $A$ is bounded.

(A.2) For each commodity $l^{\prime} \in \mathcal{L}$, there exists a household $h \in \mathcal{H}$ whose preferences are strictly monotonic with respect to $x_{l}^{h}$; that is, for every $x^{h} \in X^{h}$, there exists $\hat{x}^{h} \in X^{h}$ such that $\hat{x}_{l^{\prime}}^{h}>x_{l^{\prime}}^{h}, \hat{x}_{l}^{h}=x_{l}^{h}$ for all $l \in \mathcal{L} \backslash\left\{l^{\prime}\right\}$ and $\hat{x}^{h} \succ_{h} x^{h}$.

The foregoing is assumed throughout without reminder.

\footnotetext{
${ }^{10}$ Our real economy in this section is the same as in Dehez et al. (2003). We refer the reader to that paper for proofs of the results mentioned in this section.

11 In Sections 5 and 6, treating time and uncertainty explicitly, the commodity space will be $\mathbb{R}^{L N}$, with coordinates (commodities at date events) indexed by $l s_{t} \in \mathcal{L} \times \mathcal{N}$.

${ }^{12}$ A vector $v$ is a column vector; its transpose $v^{\top}$ is a row vector. Vector inequalities are $\geq,>, \gg$.
} 


\subsection{Pricing rules}

To define "pricing rules" we need some more notation. Write $\partial Y^{j}:=\left\{y^{j} \in Y^{j} \mid \exists\right.$ $\left.\hat{y}^{j} \in Y^{j}, \hat{y}^{j} \gg y^{j}\right\}$ for the "weakly efficient boundary" of the production set $Y^{j} ; \iota^{\top}:=$ $(1, \ldots, 1) \in \mathbb{R}^{L}$ for the unit vector in $\mathbb{R}^{L} ; \Delta^{L}=\Delta:=\left\{v \in \mathbb{R}_{+}^{L} \mid \iota^{\top} v=1\right\}$ for the unit simplex in $\mathbb{R}^{L} ; S^{L}=S:=\left\{v \in \mathbb{R}^{L} \mid \iota^{\top} v=1\right\}$ for the hyperplane in $\mathbb{R}^{L}$ containing $\Delta$; and $p \in \mathbb{R}^{L}$ for a price vector.

Definition 1 A pricing rule for firmj is a correspondence $\varphi^{j}: \partial Y^{j} \times A \times \Delta \rightarrow \mathrm{S}$ that, given the market data $(\bar{a}, \bar{p})$, assigns to each production plan $y^{j} \in \partial Y^{j}$ a set of prices $\varphi^{j}\left(y^{j} ; \bar{a}, \bar{p}\right) \in S$.

A pricing rule is a correspondence associating with every technologically efficient production plan for a firm the set of price vectors at which the firm would be willing to supply that production bundle. To give content to that abstract definition, consider first a firm endowed with a smooth convex technology and maximizing profits at given prices. Its equilibrium condition ("prices equal marginal costs" in the simplest cases) requires that prices correspond, for any point $y^{j} \in \partial Y^{j}$, to the vector normal to $Y^{j}$ at $y^{j}$ (say $N^{j}\left(y^{j}\right)$ ); indeed, by definition of the normal vector, $p^{j} \in N^{j}\left(y^{j}\right)$ implies $p^{j^{\top}} y^{j} \geq p^{j^{\top}} \hat{y}^{j}$ for all $\hat{y}^{j} \in Y^{j}$. If the production set is convex, but not smooth, the normal cone to $y^{j}$ at $Y^{j}$ collects all the vectors $p^{j}$ for which $p^{j^{\top}} y^{j} \geq p^{j^{\top}} \hat{y}^{j}, \forall \hat{y}^{j} \in Y^{j}$; it may still be denoted $N^{j}\left(y^{j}\right)$. In this simple case, the pricing rule is independent of market data and given by: ${ }^{13}$

$$
\varphi^{j}\left(y^{j}\right)=\left\{p^{j} \in S \mid p^{j} \in N^{j}\left(y^{j}\right)\right\} .
$$

By definition, $N^{j}\left(y^{j}\right)$ is a cone with vertex $\{0\}$. It defines relative prices. The restriction “ $p^{j} \in S^{\text {” }}$ is one convenient normalization among many. For a set $Y^{j}$ with free disposal, the normal cone at $y^{j} \in \partial Y^{j}$ is a collection of non-negative vectors.

\subsection{Equilibrium under pricing rules}

The more interesting application based upon imperfect competition is developed in Section 2.4. Before turning to that, it is appropriate to complete the abstract theory of equilibrium under pricing rules.

Definition 2 An equilibrium under pricing rules consists of an allocation $\bar{a} \in \mathbb{R}^{(H+J) L}$ and $a$ price vector $\bar{p} \in \Delta$ such that:

(E.1) For each $h, \bar{x}^{h}$ is $\succeq_{h}$-maximal in the budget set $B^{h}(\bar{a}, \bar{p})=$ $\left\{x^{h} \in X^{h} \mid \bar{p}^{\top} x^{h} \leq \bar{p}^{\top} e^{h}+\sum_{j} \theta^{h j} \bar{p}^{\top} \bar{y}^{j}\right\}$.

(E.2) For each $j, \bar{p} \in \varphi^{j}\left(\bar{y}^{j} ; \bar{a}, \bar{p}\right)$.

(E.3) $\sum_{h \in \mathcal{H}} \bar{x}^{h}=\sum_{h \in \mathcal{H}} e^{h}+\sum_{j \in \mathcal{J}} \bar{y}^{j}$.

Thus, the standard definition is modified: profit maximization by firms is replaced by the condition that market prices belong to the pricing rule of every firm. In the special case

\footnotetext{
13 This way of describing competitive behavior is one escape from the contradiction between price setting and competition advanced in Arrow (1959).
} 
where $\varphi^{j}\left(y^{j} ; \bar{a}, \bar{p}\right) \equiv N^{j}\left(y^{j}\right)$, Definition 2 corresponds to the definition of competitive equilibrium.

Existence requires that pricing rules be "well-behaved." Not surprisingly, the following conditions emerge, $j \in \mathcal{J}$ :

(P.1) The correspondence $\varphi^{j}$ is upper hemi-continuous and compact, convex, and nonempty valued at $\left(y^{j} ; \bar{a}, \bar{p}\right)$.

(P.2) For all $p^{j} \in \varphi^{j}\left(y^{j} ; \bar{a}, \bar{p}\right), p^{j^{\top}} y^{j} \geq 0$.

The first postulate is required to invoke Kakutani's fixed-point theorem. The second is crucial in proving that a fixed point is an equilibrium, and is also needed to guarantee non-negative property incomes. ${ }^{14}$

Theorem 1 For the economy of Section 2.1, under P.1 and P.2, there exists an equilibrium with pricing rules.

\subsection{Equilibrium under imperfect competition à la Negishi}

A more interesting application deals with imperfect competition. The starting point comes from Negishi $(1961,1972)$, who considers "perceived inverse demand functions" defined, at market signals $(\bar{a}, \bar{p})$, by

$$
\pi^{j}\left(y^{j} ; \bar{a}, \bar{p}\right)=\bar{p}+H^{j}(\bar{a}, \bar{p})\left(y^{j}-\bar{y}^{j}\right),
$$

with $H^{j}(\bar{a}, \bar{p})$ a negative semi-definite (NSD) matrix. In words: the right-hand side of (1) gives the prices at which the firm expects to be able to trade the quantities $y^{j}$, when the market data are $(\bar{a}, \bar{p})$. We make the following assumptions.

(A.3) For each $j \in \mathcal{J}$, the mapping $(\bar{a}, \bar{p}) \rightarrow H^{j}(\bar{a}, \bar{p})$ is continuous, and the matrix $H^{j}(\bar{a}, \bar{p})$ is NSD for $(\bar{a}, \bar{p}) \in A \times \Delta$.

(A.4) For each $j \in \mathcal{J}$, the matrix $H^{j}(\bar{a}, \bar{p})^{\top}$ owns at least one row with zero entries, identically in $(\bar{a}, \bar{p}){ }^{15}$

The associated profits are

$$
y^{j^{\top}}\left(\bar{p}-H^{j}(\bar{a}, \bar{p}) \bar{y}^{j}\right)+y^{j^{\top}} H^{j}(\bar{a}, \bar{p}) y^{j} .
$$

In the real (i.e. non-monetary) economy, it is natural to take $\bar{p} \in \Delta$, and to impose that equations (1) be homogeneous of degree 1 in the overall price level $\iota^{\top} \bar{p}$; that is, for $\bar{p} \in \Delta$ and for all $k>0$ :

$$
\pi^{j}\left(y^{j} ; \bar{a}, k \bar{p}\right)=k \bar{p}+k H^{j}(\bar{a}, \bar{p})\left(y^{j}-\bar{y}^{j}\right) .
$$

\footnotetext{
${ }^{14}$ Since $\{0\} \in Y^{j},(\mathrm{P} .2)$ is always satisfied when $\varphi^{j}\left(y^{j} ; \bar{a}, \bar{p}\right)=N^{j}\left(y^{j}\right)$.

${ }^{15}$ Assumption A.4 justifies the restriction of the pricing rule to production plans in $\partial Y^{j}$. Indeed, under strictly positive prices a production plan in the interior of $Y^{j}$ cannot be profit maximizing because increasing $y_{l}^{j}$, where $l$ corresponds to a zero row of $H^{j}(\bar{a}, \bar{p})^{\top}$, increases the profits of firm $j$.
} 
We will make such an assumption in Section 6, when we study the indeterminacy and neutrality of the overall price level in a monetary economy.

In equilibrium, the producer will choose a production bundle consistent with the market data, $y^{j}=\bar{y}^{j}$. First-order conditions (FOC) for maximal profits at $\bar{y}^{j}$ require the existence of a vector $q^{j}$ in the normal cone $N^{j}\left(\bar{y}^{j}\right)$ such that ${ }^{16,17}$

$$
\bar{p}+H^{j}(\bar{a}, \bar{p})^{\top} \bar{y}^{j}-q^{j}=0 .
$$

Example 1 In a typical illustration, the firm has competitive demand perceptions for most commodities, inducing zeros in the corresponding rows and columns of the matrix $H$. If the price of commodity $l$ is unaffected by $y^{j}$, then row $l$ of the matrix $H^{j}(\bar{a}, \bar{p})$ has zero entries; if the level of $y_{l}^{j}$ does not affect prices, then column $l$ of the matrix $H^{j}(\bar{a}, \bar{p})$ has zero entries.

In the simplest case of a single output $l$ produced from a single input $k$, and competitive demand perceptions for the input (as well as for commodities not used in the production process), FOC (2) boil down to

$$
\begin{aligned}
\bar{p}_{l}+\bar{y}_{l}^{j} H_{l l}^{j}(\bar{a}, \bar{p}) & =q_{l}^{j}, \\
\bar{p}_{k} & =q_{k}^{j},
\end{aligned}
$$

which in turn implies

$$
\bar{p}_{l}\left(1+H_{l l}^{j}(\bar{a}, \bar{p}) \frac{\bar{y}_{l}^{j}}{\bar{p}_{l}}\right)=\bar{p}_{k} \frac{q_{l}^{j}}{q_{k}^{j}}=\bar{p}_{k} M R T_{l k}\left(\bar{y}^{j}\right),
$$

the well-known formula that output price times one plus the output elasticity of price equals marginal cost. FOC (2) hold with any number of inputs and outputs, whose prices may or may not react to the firm's choice of $y^{j}$.

The presence of $(\bar{a}, \bar{p})$ as determinants of the matrix $H^{j}(\bar{a}, \bar{p})$ reflects the obvious dependence of demand perceptions at the firm level upon the market data. Thus, the perceived elasticity of demand for output $l$ of firm $j$ is apt to depend upon, for instance, the market prices of substitutes $l^{\prime}$ not supplied by firm $j$; or the aggregate supply of commodity $l$ by competitors of $j$ at a price $\bar{p}_{l}$.

Consider equation (2). Because $q^{j} \in N^{j}\left(\bar{y}^{j}\right)$, it can be written as $\lambda \bar{q}^{j}$ with $\lambda \geq 0$ and $\bar{q}^{j} \in \Delta$. Because $\pi^{j}\left(\bar{y}^{j} ; \bar{a}, \bar{p}\right)=\bar{p}, \lambda$ is equal to $1+\iota^{\top} H^{j}(\bar{a}, \bar{p})^{\top} \bar{y}^{j}$. Equation (2) may therefore be rewritten as

$$
\bar{p}=\left(1+\iota^{\top} H^{j}(\bar{a}, \bar{p})^{\top} \bar{y}^{j}\right) \bar{q}^{j}-H^{j}(\bar{a}, \bar{p}) \bar{y}^{j} .
$$

\footnotetext{
${ }^{16}$ See Clarke (1983), proposition 2.3.1 and corollary to proposition 2.4.3. With $H^{j}(\bar{a}, \bar{p}) \operatorname{NSD}$ and $Y^{j}$ convex, the FOC are necessary and sufficient for a global maximum.

${ }^{17}$ In the special case where the production possibility set $Y^{j}$ is described by a differentiable transformation function $F^{j}: \mathbb{R}^{L} \rightarrow \mathbb{R}$ with the property that $F^{j}\left(y^{j}\right) \leq 0$ if and only if $y^{j} \in Y^{j}$, the usual Kuhn-Tucker conditions deliver FOC (2) with $q^{j}$ equal to a non-negative multiple of $\partial F^{j}\left(\bar{y}^{j}\right)$. The vector $q^{j}$ is therefore related to the marginal rate of transformation of good $l$ for good $k$ at $\bar{y}^{j}$, which equals $\partial_{y_{l}^{j}} F^{j}\left(\bar{y}^{j}\right) / \partial_{y_{k}^{j}} F^{j}\left(\bar{y}^{j}\right)$.
} 
From this it would be tempting to define the pricing rule $\bar{\varphi}^{j}$ at $\bar{y}^{j}$ by

$$
\begin{aligned}
\bar{\varphi}^{j}\left(\bar{y}^{j} ; \bar{a}, \bar{p}\right) & =\left\{p^{j} \in \mathbb{R}^{L} \mid \exists \bar{q}^{j} \in N^{j}\left(\bar{y}^{j}\right) \cap \Delta:\right. \\
p^{j} & \left.=\left(1+\iota^{\top} H^{j}(\bar{a}, \bar{p})^{\top} \bar{y}^{j}\right) \bar{q}^{j}-H^{j}(\bar{a}, \bar{p}) \bar{y}^{j}\right\} .
\end{aligned}
$$

However, $\lambda=1+\iota^{\top} H^{j}(\bar{a}, \bar{p})^{\top} \bar{y}^{j}$ may be negative, in which case $\bar{p} \in \bar{\varphi}^{j}\left(\bar{y}^{j} ; \bar{a}, \bar{p}\right)$ does not imply that the firm maximizes profits at $\bar{y}^{j}$ given market data $(\bar{a}, \bar{p})$.

To avoid this problem, we define the pricing rule $\varphi^{j}$ at $\bar{y}^{j}$ by

$$
\begin{aligned}
\varphi^{j}\left(\bar{y}^{j} ; \bar{a}, \bar{p}\right) & =\left\{p^{j} \in \mathbb{R}^{L} \mid \exists \bar{q}^{j} \in N^{j}\left(\bar{y}^{j}\right) \cap \Delta:\right. \\
p^{j} & \left.=\max \left(0,1+\iota^{\top} H^{j}(\bar{a}, \bar{p})^{\top} \bar{y}^{j}\right) \bar{q}^{j}-\frac{H^{j}(\bar{a}, \bar{p})^{\top} \bar{y}^{j}}{\max \left(1,-\iota^{\top} H^{j}(\bar{a}, \bar{p})^{\top} \bar{y}^{j}\right)}\right\} .
\end{aligned}
$$

When $1+\iota^{\top} H^{j}(\bar{a}, \bar{p})^{\top} \bar{y}^{j} \geq 0$, the first-order condition (2) holds whenever $\bar{p} \in \varphi^{j}\left(\bar{y}^{j} ; \bar{a}, \bar{p}\right)$. Otherwise, $p^{j}=H^{j}(\bar{a}, \bar{p})^{\top} \bar{y}^{j} / \iota^{\top} H^{j}(\bar{a}, \bar{p})^{\top} \bar{y}^{j}$ for all $p^{j} \in \varphi^{j}\left(\bar{y}^{j} ; \bar{a}, \bar{p}\right)$. Because $H^{j}(\bar{a}, \bar{p})^{\top}$ contains at least one vanishing row, the corresponding element of $p^{j}$ is set equal to zero. Under Assumption A.2, $\bar{p}_{l}=0$ is impossible at equilibrium, implying that the adjustment to the FOC does not matter in equilibrium.

We must also define the pricing rule for $y^{j} \neq \bar{y}^{j}$. Because this case is incompatible with equilibrium, we may use any specification that satisfies P.1 and P.2. The simplest such specification is the following:

$$
\begin{aligned}
\varphi^{j}\left(y^{j} ; \bar{a}, \bar{p}\right) & =\left\{p^{j} \in \mathbb{R}^{L} \mid \exists \bar{q}^{j} \in N^{j}\left(y^{j}\right) \cap \Delta:\right. \\
p^{j} & \left.=\max \left(0,1+\iota^{\top} H^{j}(\bar{a}, \bar{p})^{\top} y^{j}\right) \bar{q}^{j}-\frac{H^{j}(\bar{a}, \bar{p})^{\top} y^{j}}{\max \left(1,-\iota^{\top} H^{j}(\bar{a}, \bar{p})^{\top} y^{j}\right)}\right\} .
\end{aligned}
$$

This amounts to using at $y^{j} \neq \bar{y}^{j}$ the matrix of partial derivatives $H^{j}(\bar{a}, \bar{p})$ characterizing demand perceptions at $(\bar{a}, \bar{p})$.

Lemma 1 Under Assumption A.3, for $\left(y^{j} ; \bar{a}, \bar{p}\right)$ in a compact set, the pricing rule (4) satisfies P.1 and P.2.

Lemma 1 allows Dehez et al. (2003) to invoke Theorem 1 and state an existence theorem, which we extend to kinky perceived demands in Section 3.

\section{Real equilibria under kinky demands perceived by firms}

\subsection{Pricing rules}

Extending the model of Section 2 to kinky perceived demands looks at first sight straightforward: just replace the demand function (1) by a function with a kink at $\bar{y}^{j}$; and draw the implications for profit maximization! 
Example 2 This natural intuition is readily illustrated by an extension of Example 1 to asymmetrical perceived demand elasticities. Assume that the firm perceives alternative demand slopes $H_{l l}^{j+}(\bar{a}, \bar{p})$ and $H_{l l}^{j-}(\bar{a}, \bar{p})$ applicable, respectively, to increases $\left(d y_{l}^{j}>0\right)$ and decreases $\left(d y_{l}^{j}<0\right)$ of $\bar{y}_{l}^{j}$. That is, the firm holds a kinky demand perception at $\bar{y}^{j}$. For the objective function of the firm to be concave, it must be the case that $H_{l l}^{j+}(\bar{a}, \bar{p}) \bar{y}_{l}^{j} \leq H_{l l}^{j-}(\bar{a}, \bar{p}) \bar{y}_{l}^{j}$. Under that condition, it is readily verified that the FOC are given by the inequalities

$$
\bar{p}_{l}\left(1+H_{l l}^{j+}(\bar{a}, \bar{p}) \frac{\bar{y}_{l}^{j}}{\bar{p}_{l}}\right) \leq \bar{p}_{k} \frac{q_{l}^{j}}{q_{k}^{j}} \leq \bar{p}_{l}\left(1+H_{l l}^{j-}(\bar{a}, \bar{p}) \frac{\bar{y}_{l}^{j}}{\bar{p}_{l}}\right),
$$

which generalize naturally the equality (3). Under (5), marginal revenue of the last unit of $\bar{y}_{l}^{j}$ covers marginal cost, possibly with a profit margin; but marginal revenue of an extra unit does not.

Beyond the simplicity of the intuition and of the example, a number of new technical issues arise in extending (5) to the general case (many goods and abstract technology). These are handled through Lemmas 3 and 4, proved in the Appendix. The resulting pricing rule (11) relates to (4) in basically the same way that (5) relates to (3). Lemma 5 parallels Lemma 1.

In order to study kinks in $\mathbb{R}^{L}$, note first that $\mathbb{R}^{L}$ has $2^{L}$ orthants (thus, 2 for the line $\mathbb{R}$, 4 for the plane $\mathbb{R}^{2}, 8$ for $\mathbb{R}^{3}$ etc.). Index these orthants by the $L$-vectors $s \in \mathcal{S}$, where

$$
\mathcal{S}=\left\{s \in \mathbb{R}^{L} \mid \text { for every } l \in \mathcal{L} \text {, either } s_{l}=+1 \text { or } s_{l}=-1\right\} .
$$

An orthant with origin at $0 \in \mathbb{R}^{L}$, denoted $\mathbb{R}^{L}(0, s)$, is then defined by

$$
\mathbb{R}^{L}(0, s)=\left\{v \in \mathbb{R}^{L} \mid \text { for every } l \in \mathcal{L}, s_{l}\left(v_{l}-0\right) \geq 0\right\} .
$$

A piecewise linear $L$-dimensional demand function with a kink at $\bar{y}^{j}$ thus has (potentially) $2^{L}$ different derivatives, one for each displaced orthant of $\mathbb{R}^{L}$ with origin at $\bar{y}^{j}$. We define

$$
\mathbb{R}^{L}\left(\bar{y}^{j}, s\right)=\left\{y \in \mathbb{R}^{L} \mid \text { for every } l \in \mathcal{L}, s_{l}\left(y_{l}-\bar{y}_{l}\right) \geq 0\right\} .
$$

At an interior point $y^{j}$ of a displaced orthant defined by a sign vector $s$, the perceived demands are linear, with partial derivatives

$$
\frac{\partial \pi_{k}^{j}}{\partial y_{l}^{j}}\left(y^{j} ; \bar{a}, \bar{p}\right)=H_{k l}^{j s}(\bar{a}, \bar{p})
$$

resulting in a matrix $H^{j s}(\bar{a}, \bar{p})$. Using these definitions, we extend (1) to perceived demand functions with a kink at $\bar{y}^{j}$, defined by

$$
\pi^{j}\left(y^{j} ; \bar{a}, \bar{p}\right)=\bar{p}+H^{j s}(\bar{a}, \bar{p})\left(y^{j}-\bar{y}^{j}\right), \quad s \in \mathcal{S}, y^{j} \in \mathbb{R}^{L}\left(\bar{y}^{j}, s\right) .
$$

For $\bar{y}^{j}$ to be a profit-maximizing vector under the piecewise linear perceived demands (6), $2^{L}$ inequalities must be satisfied, extending the two inequalities in (5); namely, the $2^{L}$ directional derivatives of $y^{j^{\top}} \pi^{j}\left(y^{j} ; \bar{a}, \bar{p}\right)$ in the $2^{L}$ displaced orthants $\mathbb{R}^{L}\left(\bar{y}^{j}, s\right)$ 
correspond to non-increasing profits. That is, for all $s \in \mathcal{S}$, for all $d y^{j}$ such that $\bar{y}^{j}+$ $d y^{j} \in \mathbb{R}^{L}\left(\bar{y}^{j}, s\right)$

$$
d y^{j^{\top}} \bar{p}+\left(\bar{y}^{j}+d y^{j}\right)^{\top} H^{j s}(\bar{a}, \bar{p}) d y^{j} \leq 0 .
$$

The next lemma shows that there is no need to specify $2^{L}$ matrices $H^{j s}(\bar{a}, \bar{p})$. Once the matrix corresponding to the sign vector with every component +1 , denoted $H^{j+}(\bar{a}, \bar{p})$, and the matrix corresponding to the sign vector with every component -1 , denoted $H^{j-}(\bar{a}, \bar{p})$, are given, piecewise linearity of the perceived inverse demand function $\pi^{j}$ implies that column $l$ of $H^{j s}(\bar{a}, \bar{p})$ equals column $l$ of $H^{j-}(\bar{a}, \bar{p})$ if $s_{l}=-1$ and equals column $l$ of $H^{j+}(\bar{a}, \bar{p})$ if $s_{l}=+1$.

Lemma 2 If $\pi^{j}$ is a piecewise linear perceived inverse demand function with a kink at $\bar{y}^{j}$ given market data $(\bar{a}, \bar{p})$, then

$$
H_{\cdot l}^{j s}(\bar{a}, \bar{p})= \begin{cases}H_{\cdot l}^{j-}(\bar{a}, \bar{p}) & \text { if } s_{l}=-1, \\ H_{\cdot l}^{j+}(\bar{a}, \bar{p}) & \text { if } s_{l}=+1 .\end{cases}
$$

Proof: See Appendix.

Motivated by Lemma 2, our primitives are $H^{j-}(\bar{a}, \bar{p}), H^{j+}(\bar{a}, \bar{p})$, the NSD matrices of demand derivatives applicable, respectively, to negative and positive adjustments $d y^{j}$. Accordingly, every matrix $H^{j s}(\bar{a}, \bar{p})$ consists of columns borrowed from either $H^{j-}(\bar{a}, \bar{p})$ or $H^{j+}(\bar{a}, \bar{p})$, depending upon the sign vector, $s$. Moreover, for $s \in \mathcal{S}$, we shall require the matrices $H^{j s}(\bar{a}, \bar{p})$ to be NSD.

Lemma 3 provides the necessary and sufficient conditions for $\bar{y}^{j}$ to be a profit maximizing production plan given market data $(\bar{a}, \bar{p})$.

Lemma 3 The production plan $\bar{y}^{j}$ maximizes profits given market data $(\bar{a}, \bar{p})$ if and only if for every $s \in \mathcal{S}$, there exists $q^{j s} \in N^{j}\left(\bar{y}^{j}\right)$ and $\mu^{j s} \in \mathbb{R}^{L}(0, s)$ such that ${ }^{18}$

$$
\bar{p}+H^{j s}(\bar{a}, \bar{p})^{\top} \bar{y}^{j}=q^{j s}-\mu^{j s} .
$$

Proof: See Appendix.

Using the necessary and sufficient conditions of Lemma 3, we can derive the following simpler conditions, which we will prove to be sufficient.

Lemma 4 The production plan $\bar{y}^{j}$ maximizes profits given market data $(\bar{a}, \bar{p})$ if there exists $q^{j} \in N^{j}\left(\bar{y}^{j}\right)$ such that the following pair of inequalities is satisfied:

$$
\begin{aligned}
& \bar{p} \geq q^{j}-H^{j-}(\bar{a}, \bar{p})^{\top} \bar{y}^{j}, \\
& \bar{p} \leq q^{j}-H^{j+}(\bar{a}, \bar{p})^{\top} \bar{y}^{j} .
\end{aligned}
$$

${ }^{18}$ Observe that $\mu^{j s} \in \mathbb{R}^{L}(0, s)$ if and only if $\mu_{l}^{j s} s_{l} \geq 0$, for all $l \in \mathcal{L}$. 
Proof: See Appendix.

Notice that existence of solutions to (7)-(8) requires

$$
H^{j+}(\bar{a}, \bar{p})^{\top} \bar{y}^{j} \leq H^{j-}(\bar{a}, \bar{p})^{\top} \bar{y}^{j} .
$$

This condition is implied by concavity of the objective function of the firm.

Using (9) and building upon (4) it would be natural to define the pricing rule as

$$
\begin{aligned}
& \bar{\varphi}^{j}\left(y^{j} ; \bar{a}, \bar{p}\right)=\left\{p^{j} \in S \mid \exists \bar{q}^{j} \in N^{j}\left(y^{j}\right) \cap \Delta\right. \text { such that } \\
& \max \left(0,1+\iota^{\top} \bar{H}^{j}(\bar{a}, \bar{p})^{\top} y^{j}\right) \bar{q}^{j}-\frac{H^{j-}(\bar{a}, \bar{p})^{\top} y^{j}}{\max \left(1,-\iota^{\top} \bar{H}^{j}(\bar{a}, \bar{p})^{\top} y^{j}\right)} \leq p^{j} \leq \\
& \left.\max \left(0,1+\iota^{\top} \bar{H}^{j}(\bar{a}, \bar{p})^{\top} y^{j}\right) \bar{q}^{j}-\frac{H^{j+}(\bar{a}, \bar{p})^{\top} y^{j}}{\max \left(1,-\iota^{\top} \bar{H}(\bar{a}, \bar{p})^{\top} y^{j}\right)}\right\},
\end{aligned}
$$

where $\bar{H}^{j}=\left(H^{j-}+H^{j+}\right) / 2 .{ }^{19}$ The problem of this specification is that $\bar{\varphi}^{j}$ is empty valued if it is not the case that $H^{j+}(\bar{a}, \bar{p})^{\top} y^{j} \leq H^{j-}(\bar{a}, \bar{p})^{\top} y^{j}$, which may occur for some $y^{j} \in \partial Y^{j}$. To solve this problem, we adapt the pricing rule for $y^{j} \neq \bar{y}^{j}$ by letting $H^{j+}$ and $H^{j-}$ depend on $y^{j}$. We denote the resulting matrices by $H^{j+}\left(y^{j} ; \bar{a}, \bar{p}\right)$ and $H^{j-}\left(y^{j} ; \bar{a}, \bar{p}\right)$, imposing only that $H^{j s}\left(y^{j} ; \bar{a}, \bar{p}\right)$ is NSD for all $s \in \mathcal{S}$ (Assumption A.3' below), that $H^{j-}\left(\bar{y}^{j} ; \bar{a}, \bar{p}\right)=H^{j-}(\bar{a}, \bar{p})$ and $H^{j+}\left(\bar{y}^{j} ; \bar{a}, \bar{p}\right)=H^{j+}(\bar{a}, \bar{p})$, and

$$
H^{j+}\left(y^{j} ; \bar{a}, \bar{p}\right)^{\top} y^{j} \leq H^{j-}\left(y^{j} ; \bar{a}, \bar{p}\right)^{\top} y^{j} .
$$

Because in equilibrium $y^{j}=\bar{y}^{j}$, the specification of these matrices for $y^{j} \neq \bar{y}^{j}$ is immaterial. We obtain the following definition of the pricing rule:

$$
\begin{aligned}
& \varphi^{j}\left(y^{j} ; \bar{a}, \bar{p}\right)=\left\{p^{j} \in S \mid \exists \bar{q}^{j} \in N^{j}\left(y^{j}\right) \cap \Delta\right. \text { such that } \\
& \max \left(0,1+\iota^{\top} \bar{H}^{j}\left(y^{j} ; \bar{a}, \bar{p}\right)^{\top} y^{j}\right) \bar{q}^{j}-\frac{H^{j-}\left(y^{j} ; \bar{a}, \bar{p}\right)^{\top} y^{j}}{\max \left(1,-\iota^{\top} \bar{H}{ }^{j}\left(y^{j} ; \bar{a}, \bar{p}\right)^{\top} y^{j}\right)} \leq p^{j} \leq \\
& \left.\max \left(0,1+\iota^{\top} \bar{H}^{j}\left(y^{j} ; \bar{a}, \bar{p}\right)^{\top} y^{j}\right) \bar{q}^{j}-\frac{H^{j+}\left(y^{j} ; \bar{a}, \bar{p}\right)^{\top} y^{j}}{\max \left(1,-\iota^{\top} \bar{H}^{j}\left(y^{j} ; \bar{a}, \bar{p}\right)^{\top} y^{j}\right)}\right\} .
\end{aligned}
$$

It is readily verified that, given (10), and whether or not $1+\iota^{\top} \bar{H}^{j}\left(y^{j} ; \bar{a}, \bar{p}\right)^{\top} y^{j} \geq 0$, the left-hand side vector is less than or equal to the right-hand side vector; and the elements of these vectors sum up to no more than one on the left and no less than one on the right. Therefore, (11) admits solutions with $\iota^{\top} p=1$; but that property must be imposed through the additional side condition $p^{j} \in S$ : otherwise not all solutions of (11) need satisfy it.

When $H^{j-}(\cdot) \equiv H^{j+}(\cdot)$, (11) reduces to (4); when $H^{j-}(\cdot) \equiv H^{j+}(\cdot) \equiv 0$, the pricing rule defines competitive behavior (prices in $N^{j}\left(y^{j}\right)$, hence sustaining $y^{j}$ as a

\footnotetext{
${ }^{19}$ Any $\bar{H}^{j}$ satisfying $H^{j+}(\cdot)^{\top} y^{j} \leq \bar{H}^{j}(\cdot)^{\top} y^{j} \leq H^{j-}(\cdot)^{\top} y^{j}$ would do; there is no loss of generality in using the mean of $H^{j+}$ and $H^{j-}$, but there is an economy of notation.
} 
profit-maximizing production plan at given prices). Thus, (11) encompasses a hierarchy of market situations.

\subsection{Existence of equilibrium}

An equilibrium under pricing rules is still defined by Definition 2. To prove existence, we must extend Assumptions A.3 and A.4 to allow for kinks.

(A.3') For each $j \in \mathcal{J}$, the mapping $\left(H^{j-}, H^{j+}\right): \partial Y^{j} \times A \times \Delta \rightarrow \mathbb{R}^{2 L^{2}}$, which maps $\left(y^{j}, \bar{a}, \bar{p}\right)$ to $\left(H^{j-}\left(y^{j} ; \bar{a}, \bar{p}\right), H^{j+}\left(y^{j} ; \bar{a}, \bar{p}\right)\right)$, is continuous and the matrices $H^{j-}\left(y^{j} ; \bar{a}, \bar{p}\right), H^{j+}\left(y^{j} ; \bar{a}, \bar{p}\right)$, as well as the derived matrices $H^{j s}\left(y^{j} ; \bar{a}, \bar{p}\right)$, $s \in \mathcal{S}$, are NSD. It holds that $\left(H^{j-}\left(\bar{y}^{j} ; \bar{a}, \bar{p}\right), H^{j+}\left(\bar{y}^{j} ; \bar{a}, \bar{p}\right)\right)=\left(H^{j-}(\bar{a}, \bar{p})\right.$, $\left.H^{j+}(\bar{a}, \bar{p})\right) .^{20}$

(A. $\left.4^{\prime}\right)$ For each $j \in \mathcal{J}$, there exists at least one commodity $\hat{l}(j)$ such that row $\hat{l}(j)$ of both matrices $H^{j-}\left(y^{j} ; \bar{a}, \bar{p}\right)^{\top}$ and $H^{j+}\left(y^{j} ; \bar{a}, \bar{p}\right)^{\top}$ consists of zero entries, identically in $\left(y^{j}, \bar{a}, \bar{p}\right)$.

(A.5) For each $j \in \mathcal{J}$, for each $y^{j} \in \partial Y^{j}$, the inequalities

$$
H^{j+}\left(y^{j} ; \bar{a}, \bar{p}\right)^{\top} y^{j} \leq H^{j-}\left(y^{j} ; \bar{a}, \bar{p}\right)^{\top} y^{j}
$$

hold.

Lemma 5 Under Assumptions A.3' and A.5, for $\left(y^{j}, \bar{a}, \bar{p}\right)$ in a compact set, the pricing rule (11) satisfies P.1 and P.2.

Proof: See Appendix.

Lemma 5 plays a central role in our analysis. It allows us to apply Theorem 2.3 and to state:

Theorem 2 For the economy of Section 3.1, under Assumptions A.3', A.4', and A.5, there exists an equilibrium with pricing rules as defined by (11); that is, with profit maximization under possibly kinky perceived demand functions.

\section{Real equilibria under kinky demands perceived by unions}

\subsection{Background}

As mentioned cursorily in Section 1.2, the published literature discusses several formulations of the wage determination problem when labor is organized as unions. One important distinction concerns the process itself, either bargaining between firms and unions, or wage-setting by unions followed by hiring decisions of the firms. In either case, the objective functions of the unions must be specified.

\footnotetext{
${ }^{20}$ We impose assumptions on $\left(H^{j-}, H^{j+}\right)$ as functions defined on $\partial Y^{j} \times A \times \Delta$. We could instead have taken $\left(H^{j-}, H^{j+}\right)$ as defined on $A \times \Delta$ as a primitive, and then defined them on $Y^{j} \times A \times \Delta$ by taking appropriate extensions and projections.
} 
Bargaining models are typically asymmetrical Nash, with a parameter measuring the relative bargaining powers of employers and unions (see e.g. Arnsperger and de la Croix 1993; Licandro 1995; Bénassy 2002). These authors eschew the "objective function" issue by assuming identical workers. We do not follow the bargaining path, because our purpose is to explore kinky demand curves perceived by unions.

There is significant literature on wage-setting unions, dating as far back as Dunlop (1944). Typically, that literature deals with objective demand curves obtained analytically in simplified models. Dunlop, or more recently Hart (1982), equates the union's objective to the aggregate wage bill. Some authors endow unions outright with a utility function; (e.g. Oswald 1979; Calmfors 1982). Others assume that union members have identical preferences (e.g. Snower 1983), others still endow individual workers with market power (e.g. Blanchard and Kiyotaki 1987; Bénassy 2002). Blair and Crawford (1984) and Farber (1986) handle heterogeneous preferences through a "median voter" model, and recognize its limitations. Negishi (1979) also assumes identical preferences across union members, but relies on perceived demand functions with kinks. ${ }^{21}$

The general equilibrium framework used here has both strengths and weaknesses for modeling unions and non-clearing labor markets. In a framework encompassing time and uncertainty, every agent (firm, household, or labor union) is concerned with several labor markets. We are not appraised of simplified models with objective demand curves applicable to a general multi-market framework. Existence proofs in general equilibrium theory rely on strong continuity properties. Accordingly, models with fixed labor time (working week), where workers are either employed full-time or totally inactive, are not covered by the standard theory; they are unwieldy, unless one introduces a continuum of workers. We must accordingly proceed under the restrictive assumption that aggregate employment on a specific unionized labor market is divided among union members in a continuous way. This is the price of generality, in the present context. ${ }^{22}$

\subsection{A simple example}

Before proceeding to the formal analysis, it is helpful to review a simple example, with: (i) a single type of labor supplied by the members of a wage-setting union; and (ii) member preferences represented by $C^{1}$ utility functions.

Example 3 Consider a union with $n$ members, setting the wage $w$ for a single type of labor. Aggregate employment is denoted $\ell$, and the perceived inverse demand function of the union is $w(\ell)$, with $\frac{d w}{d \ell}<0$. Each member $h$ of the union will supply a quantity of labor $\ell^{h}(\ell)$, with $\frac{d \ell^{h}}{d \ell} \geq 0$ and $\sum_{h} \ell^{h}(\ell) \equiv \ell>0$. Union member $h$ has a disposable income $y^{h}=w \ell^{h}$, and preferences represented by the mixed direct-indirect $C^{1}$ utility function $u^{h}\left(\ell^{h}, y^{h}\right)=u^{h}\left(\ell^{h}, w \ell^{h}\right)$.

\footnotetext{
${ }^{21}$ For a survey of the union-wage model, see Oswald (1985). Models with union utility or with identical members are special cases of our model. Wage bill maximization is at variance with our approach; see remark (vi) in Subsection 4.2.

${ }^{22}$ See, however, remark (iv) at the end of Subsection 4.2.
} 
The total derivative of $u^{h}$ with respect to $\ell$ is:

$$
\frac{d u^{h}}{d \ell}=\frac{\partial u^{h}}{\partial \ell^{h}} \frac{d \ell^{h}}{d \ell}+\frac{\partial u^{h}}{\partial y^{h}}\left(w \frac{d \ell^{h}}{d \ell}+\frac{d w}{d \ell} \ell^{h}\right) .
$$

A level of employment $\ell$ is Pareto-efficient for the union members if it maximizes $\sum_{h} \mu^{h} u^{h}\left(\ell^{h}, w \ell^{h}\right)$ for some positive vector of weights $\mu^{h}$. The FOC for that problem is

$$
\sum_{h} \mu^{h} \frac{d u^{h}}{d \ell}=\sum_{h} \mu^{h} \frac{\partial u^{h}}{\partial y^{h}}\left\{w \frac{d \ell^{h}}{d \ell}+\frac{\partial u^{h} / \partial \ell^{h}}{\partial u^{h} / \partial y^{h}} \frac{d \ell^{h}}{d \ell}+\frac{d w}{d \ell} \ell^{h}\right\}=0 .
$$

Because $\frac{\partial u^{h}}{\partial y^{h}}$ has arbitrary scale, the terms $\mu^{h} \frac{\partial u^{h}}{\partial y^{h}}$ are undetermined individual coefficients assigned to member incomes in the optimization problems. (In contrast, all the terms in the curly brackets are well-defined.) A union not pursuing interpersonal redistributive policies $^{23}$ behaves $a s$ if it assigned equal weights to the incomes of all its members (i.e. as if $\mu^{h} \frac{\partial u^{h}}{\partial y^{h}}$ were independent of $\left.h\right)$. In that case, using $\sum_{h} \frac{d \ell^{h}}{d \ell}=1,(12)$ may be rewritten as: ${ }^{24}$

$$
w=-\sum_{h} \frac{\partial u^{h} / \partial \ell^{h}}{\partial u^{h} / \partial y^{h}} \frac{d \ell^{h}}{d \ell}-\frac{d w}{d \ell} \ell .
$$

The first term in (13) contains the marginal rates of substitution between work and income of all union members, weighted by their stakes in marginal adjustments of aggregate employment $d \ell$. Two remarks:

(i) This is exactly the formula defining shadow prices for future state-dependent outputs supporting production plans that are Pareto-efficient for shareholders, under incomplete markets (Drèze 1974).

(ii) Marginal rates of substitution between work and income define the reservation wages of workers.

Formula (13) thus admits of a natural interpretation: union wages should correspond to average reservation wages of members, augmented with a markup reflecting the demand elasticity.

We show that (13) is analogous to (3). Let $\mathcal{H}^{k}$ be the set of members of union $k$. Write $y_{l}^{k}$ for aggregate employment $\ell$ of members of union $k, \bar{p}_{l}=\pi_{l}^{k}\left(\bar{y}^{k} ; \bar{a}, \bar{p}\right)$ for the wage ("price") $w$ and $H_{l l}^{k}(\bar{a}, \bar{p})$ for $d w / d \ell$, assuming market signals $(\bar{a}, \bar{p})$, where $\bar{y}^{k}$ is consistent with market data, so $\bar{y}_{l}^{k}=-\sum_{h \in \mathcal{H}^{k}}\left(\bar{x}_{l}^{h}-e_{l}^{h}\right)$. Furthermore, define $\lambda^{h}=-\frac{\partial u^{h} / \partial \ell^{h}}{\partial u^{h} / \partial y^{h}}$ and $\lambda^{k}=\sum_{h \in \mathcal{H}^{k}} \lambda^{h} \frac{d \ell^{h}}{d \ell}$. Then (13) may be written as

$$
\bar{p}_{l}\left(1+H_{l l}^{k}(\bar{a}, \bar{p}) \frac{\bar{y}_{l}^{k}}{\bar{p}_{l}}\right)=\lambda^{k}
$$

where the right-hand side term is equal to the average reservation wage of union $k$ members (i.e. equal to the marginal opportunity cost of $\bar{y}_{l}^{k}$ at $\left.(\bar{a}, \bar{p})\right)$.

\footnotetext{
${ }^{23}$ Others might write: a democratic union, not bestowing preferential treatment upon individual members ... ${ }^{24}$ With $\ell^{h} \geq 0, \frac{\partial u^{h}}{\partial \ell^{h}} \leq 0$ so that the first term in (13) is non-negative.
} 
Let now the union entertain kinky demand perceptions, with $\frac{d^{+} w}{d \ell}$ for $d \ell>0$ and $\frac{d^{-} w}{d \ell}$ for $d \ell<0, \frac{d^{+} w}{d \ell} \ell \leq \frac{d^{-} w}{d \ell} \ell \leq 0$. The FOC (13) should then be replaced by the pair of inequalities

$$
w+\frac{d^{+} w}{d \ell} \ell \leq-\sum_{h \in \mathcal{H}^{k}} \frac{\partial u^{h} / \partial \ell^{h}}{\partial u^{h} / \partial y^{h}} \frac{d \ell^{h}}{d \ell} \leq w+\frac{d^{-} w}{d \ell} \ell .
$$

In the notation of (14), this becomes

$$
\bar{p}_{l}\left(1+H_{l l}^{k+}(\bar{a}, \bar{p}) \frac{\bar{y}_{l}^{k}}{\bar{p}_{l}}\right) \leq \lambda^{k} \leq \bar{p}_{l}\left(1+H_{l l}^{k-}(\bar{a}, \bar{p}) \frac{\bar{y}_{l}^{k}}{\bar{p}_{l}}\right) .
$$

This formula is analogous to formula (5) above: up to the replacement of marginal cost $\bar{p}_{k} \bar{q}_{l}^{j} / \bar{q}_{k}^{j}$ by an opportunity cost $\lambda^{k}$ here.

Beyond the transparency of the example, a number of new technical issues arise in extending (15) to the general case (many goods and many unions). These are handled largely through Theorem 3, proved in the Appendix.

Before leaving this example, we add four further remarks.

(iii) The term $\frac{\partial u^{h}}{\partial \ell^{h}}$ is naturally interpreted as the "marginal disutility of work", a negative number. In the present context, it could also be interpreted as a "marginal opportunity cost" of union employment rather than other employment, also negative, but perhaps more significant.

(iv) In the case of fixed working time cum unemployment, the term $\frac{d \ell^{h}}{d \ell}$ would correspond, for an unemployed worker, to the probability of being hired under $d \ell>0$, namely $\frac{1}{n-\ell} d \ell$; for an employed worker, to the probability of being fired under $d \ell<0$, namely $\frac{1}{\ell} d \ell$. In that case, the benefit of being hired (of not being fired) is given by the finite difference $u^{h}\left(\ell^{h}, w \ell^{h}\right)-\left.u^{h}\right|_{\ell^{h}=0}$. The second term in that difference requires explanation. If it called for another job at lower wages but similar hours, the difference could be expressed as the product of the wage differential by a marginal utility of income (mean value theorem), leading back to a formula comparable to (12), hence (13).

(v) Formula (13) suggests that a union concerned with the well-being of its members should elicit their reservation wages $-\frac{\partial u^{h}}{\partial \ell^{h}} / \frac{\partial u^{h}}{\partial y^{h}}$ in order to set wages according to (13). That may seem preposterous ... yet it is perhaps a meaningful characterization of union behavior in some countries. We could adopt a less precise formulation, still consistent with (13); namely, that unions set wages on the basis of shadow prices $\lambda^{k}$ which are single valued continuous monotone functions of the members' reservation wages. This would allow for $\lambda^{k}$ depending on the reservation wages of a subset (possibly random) of union members.

(vi) It is readily seen in formula (13) that identical union members correspond to a special case of our approach. However, maximizing (with respect to $\ell$ ) the wage bill $w \ell$ would lead to the FOC $w+\frac{d w}{d \ell} \ell=0$, at variance with (13). 


\subsection{General formulation}

To formalize and generalize the foregoing, we must extend the primitives of Section 2.1 by adding unions to the set of agents and by adjusting primitive $(c)$ concerning households. We define a union $k$ by: (i) the set of labor markets on which it sets wages; (ii) its membership, consisting of households supplying such labor; (iii) the rules allocating aggregate employment among union members; (iv) the set of employment levels that are feasible for the membership; (v) the objective function of the union. We postpone item (v), which is crucial to our formulation, to Section 4.4. Items (i) and (ii) are straightforward. For item (iii), we adopt the simplest formulation; namely, fixed proportions. It is then possible to handle (iv) on the basis of upper bounds on the labor supply of individual households.

Thus, we add to the primitives of Section 2.1:

(d) A set $\mathcal{K}$ of $K$ unions indexed by $k$; each union $k$ is defined by: (i) the set $\mathcal{L}^{k} \varsubsetneqq \mathcal{L}$ of $L^{k}$ labor markets on which it sets wages, with $\mathcal{L}^{k} \cap \mathcal{L}^{k^{\prime}}=\emptyset$ for all $k^{\prime} \in \mathcal{K} \backslash\{k\}$; (ii) the set $\mathcal{H}^{k} \varsubsetneqq \mathcal{H}$ of $n^{k}$ households that are members of $k$, with $\mathcal{H}^{k} \cap \mathcal{H}^{k^{\prime}}=\emptyset$ for all $k^{\prime} \in \mathcal{K} \backslash\{k\}$; (iii) for $h \in \mathcal{H}^{k}$ and $l \in \mathcal{L}^{k}$, labor allocation coefficients $\sigma_{l}^{h k} \leq 0, \quad \sum_{h \in \mathcal{H}^{k}}$ $\sigma_{l}^{h k}=-1 ; 25$ and (iv) the vector $\hat{y}^{k} \in \mathbb{R}_{+}^{L}$ with $\hat{y}_{l}^{k}=0$ for $l \notin \mathcal{L}^{k}$ defining maximally feasible employment levels for union $k$.

We shall treat union $k$ as if it were a firm supplying labor of types $l \in \mathcal{L}^{k}$ to the rest of the economy, in quantities $y_{l}^{k} \geq 0$, with $y_{l}^{k} \leq \hat{y}_{l}^{k}$. As we will assume zero initial endowments of commodities in $\mathcal{L}^{k}$, feasibility then requires $-\sum_{h \in \mathcal{H}^{k}} x_{l}^{h}=y_{l}^{k}=\sum_{h \notin \mathcal{H}^{k}} x_{l}^{h}-\sum_{j \in \mathcal{J}} y_{l}^{j}$. For $l \notin \mathcal{L}^{k}$, we impose $y_{l}^{k}=0$. More concisely, we write $y^{k}=\left({ }^{k} y^{k}, y^{k k}\right) \in\{0\} \times \mathbb{R}_{+}^{L^{k}}$; and we partition other vectors similarly, for instance $x^{h}=\left({ }^{k} x^{h}, x^{h k}\right), p=\left({ }^{k} p, p^{k}\right)$, and so on. We define the set $Y^{k}$ by $Y^{k}=\left\{y^{k} \in \mathbb{R}_{+}^{L} \mid y^{k} \leq \hat{y}^{k}\right\}$.

Regarding households, we add the following assumption:

(A.6) (i) For each $k \in \mathcal{K}$, for all $h \in \mathcal{H}^{k}, X^{h}={ }^{k} X^{h} \times X^{h k}$, where ${ }^{k} X^{h}=\left\{\underline{x}^{h}\right\}+\mathbb{R}_{+}^{L-L^{k}}$ and $X^{h k}=\left\{x^{h} \in \mathbb{R}^{L^{k}} \mid\right.$ for $\left.l \in \mathcal{L}^{k}, \sigma_{l}^{h k} \hat{y}_{l}^{k} \leq x_{l}^{h} \leq 0\right\}$.

(ii) The preferences $\succeq_{h}$ are represented by a continuously differentiable strictly quasi-concave utility function $u^{h}: X^{h} \rightarrow \mathbb{R}$ with strictly positive partial derivatives. (iii) For all $h \in \mathcal{H}$, for each $l \in \cup_{k \in \mathcal{K}} \mathcal{L}^{k}, e_{l}^{h}=0$.

Assumption A.6.1 states that consumption sets have a product form. The product form guarantees that the labor supply determined by the union is consistent with individual consumption sets. Assumption A.6.3 normalizes the endowments of "union labor".

The vectors $y^{k}$ need not be explicitly introduced into the market clearing conditions. Equilibrium requires that, for each $k \in \mathcal{K}_{:}$(i) there exists $\bar{y}^{k} \in Y^{k}$ such that $\bar{x}_{l}^{h}=\sigma_{l}^{h k} \bar{y}_{l}^{k}$ for all $h \in \mathcal{H}^{k}$ and $l \in \mathcal{L}^{k}$; and (ii) $\bar{p} \in \varphi^{k}\left(\bar{y}^{k} ; \bar{a}, \bar{p}\right)$, where $\varphi^{k}$ denotes the pricing rule of union $k$, to be defined in (21). There is no need to explicitly introduce $\bar{y}^{k}$ in the definition of a feasible allocation $\bar{a}$, because feasibility requires, for $l \in \mathcal{L}^{k}$,

\footnotetext{
${ }^{25}$ We treat union employment levels $y_{l}^{k}$ as non-negative quantitities; household labor supplies $x_{l}^{h}$ are nonpositive quantitities; hence, $x_{l}^{h}=\sigma_{l}^{h k} y_{l}^{k}$ requires $\sigma_{l}^{h k} \leq 0$.
} 


$$
\bar{y}_{l}^{k}=-\sum_{h \in \mathcal{H}^{k}} \bar{x}_{l}^{h}
$$

so $\bar{y}^{k}$ follows from $\bar{a}$. However, the presence of unions imposes an additional condition on the allocation in equilibrium; if $\bar{a}$ is an equilibrium allocation, then for each $k \in \mathcal{K}$, there is $\bar{y}^{k} \in Y^{k}$ with, for $h \in \mathcal{H}^{k}$ and $l \in \mathcal{L}^{k}, \bar{x}_{l}^{h}=\sigma_{l}^{h k} \bar{y}_{l}^{k}$. To simplify notation, for $k \in \mathcal{K}$, we define the function $\sigma^{h k}: Y^{k} \rightarrow \mathbb{R}^{L^{k}}$ by $\sigma_{l}^{h k}\left(y^{k}\right)=\sigma_{l}^{h k} y_{l}^{k}, l \in \mathcal{L}^{k}$.

In line with Sections 2.4 and 3.1, we assume that union $k$ perceives the inverse demand functions

$$
\pi^{k}\left(y^{k} ; \bar{a}, \bar{p}\right)=\bar{p}+H^{k s}(\bar{a}, \bar{p})\left(y^{k}-\bar{y}^{k}\right), \quad s \in \mathcal{S}, y^{k} \in \mathbb{R}^{L}\left(\bar{y}^{k}, s\right) .
$$

As for firms, information on perceived demand functions can be summarized by NSD matrices $H^{k+}(\bar{a}, \bar{p})$ and $H^{k-}(\bar{a}, \bar{p})$. Regarding these we make the following assumption.

(A.7) For each $k \in \mathcal{K}$, the mapping $\left(H^{k-}, H^{k+}\right): Y^{k} \times A \times \Delta \rightarrow \mathbb{R}^{2 L^{2}}$, which maps $(\bar{a}, \bar{p})$ to $\left(H^{k-}\left(y^{k} ; \bar{a}, \bar{p}\right), H^{k+}\left(y^{k} ; \bar{a}, \bar{p}\right)\right)$, is continuous and the matrices $H^{k-}\left(y^{k} ; \bar{a}, \bar{p}\right), H^{k+}\left(y^{k} ; \bar{a}, \bar{p}\right)$, as well as the derived matrices $H^{k s}\left(y^{k} ; \bar{a}, \bar{p}\right), s \in \mathcal{S}$, are NSD. It holds that $\left(H^{k-}\left(\bar{y}^{k} ; \bar{a}, \bar{p}\right), H^{k+}\left(\bar{y}^{k} ; \bar{a}, \bar{p}\right)\right)=\left(H^{k-}(\bar{a}, \bar{p}), H^{k+}(\bar{a}, \bar{p})\right)$. For each $k \in \mathcal{K}$, for each $y^{k} \in Y^{k}$, the matrices $H^{k-}\left(y^{k} ; \bar{a}, \bar{p}\right)$ and $H^{k+}\left(y^{k} ; \bar{a}, \bar{p}\right)$ have rows and columns of zeros for all commodities $l \in \mathcal{L} \backslash \mathcal{L}^{k}$, and the inequalities

$$
H^{k+}\left(y^{k} ; \bar{a}, \bar{p}\right)^{\top} y^{k} \leq H^{k-}\left(y^{k} ; \bar{a}, \bar{p}\right)^{\top} y^{k}
$$

hold.

Write further $H^{k k s}(\bar{a}, \bar{p})$ for the $L^{k} \times L^{k}$ submatrix of $H^{k s}(\bar{a}, \bar{p})$ corresponding to the rows and columns in $\mathcal{L}^{k}$.

\subsection{Unions enhancing members' welfare}

Household $h$ solves the problem

$$
\begin{aligned}
\max _{k^{k} x^{h}} u^{h}\left({ }^{k} x^{h}, \sigma^{h k}\left(y^{k}\right)\right) \text { s.t. } & { }^{k} p^{\top k} x^{h}+p^{k^{\top}} \sigma^{h k}\left(y^{k}\right) \leq p^{\top} e^{h}+\sum_{j} \theta^{h j} p^{\top} y^{j}, \\
& \left({ }^{k} x^{h}, \sigma^{h k}\left(y^{k}\right)\right) \in X^{h} .
\end{aligned}
$$

Associated with a solution $\bar{x}^{h}=\left({ }^{k} \bar{x}^{h}, \sigma^{h k}\left(y^{k}\right)\right)$ of problem (16), we define the normalized vector of marginal utilities $\bar{\lambda}^{h}$ by $\bar{\lambda}_{l}^{h}=p_{l}, \quad l \in \mathcal{L} \backslash \mathcal{L}^{k}$, and $\bar{\lambda}_{l}^{h}=\partial_{x_{l}^{h}} u^{h}\left(\bar{x}^{h}\right) / \bar{\lambda}_{0}^{h}, \quad l \in \mathcal{L}^{k}$, where $\bar{\lambda}_{0}^{h}$ denotes the Lagrange multiplier for $h^{\prime}$ s budget constraint. The specification of $\bar{\lambda}_{l}^{h}$ for $l \in \mathcal{L} \backslash \mathcal{L}^{k}$ may not correspond to the actual normalized marginal utility of household $h$ because boundary solutions are allowed for. This does not affect our analysis, as it is only the specification of $\bar{\lambda}_{k}^{h}$ for $l \in \mathcal{L}^{k}$ that matters. Our assumptions on $u^{h}$ imply that $\bar{\lambda}_{0}^{h}>0$. It holds that $u^{h}\left(\bar{x}^{h}+d x^{h}\right) \leq u^{h}\left(\bar{x}^{h}\right)$ whenever $\bar{x}^{h}+d x^{h} \in X^{h}$ and $d x^{h^{\top}} \bar{\lambda}^{h} \leq 0$.

We may now characterize the vectors $\left(\left(\bar{x}^{h}\right)_{h \in \mathcal{H}^{k}}, \bar{y}^{k}\right)$ that are Pareto efficient from the viewpoint of union members. 
Definition 3 Consumption bundles and employment levels $\left(\left(\bar{x}^{h}\right)_{h \in \mathcal{H}^{k}}, \bar{y}^{k}\right)$ with associated prices (i.e. wages) $\pi^{k}\left(\bar{y}^{k} ; \bar{a}, \bar{p}\right)$ are Pareto efficient for $\mathcal{H}^{k}$ at market signals $(\bar{a}, \bar{p})$ if there do not exist alternative employment levels $\tilde{y}^{k} \in Y^{k}$ with associated prices $\tilde{p}=\pi^{k}\left(\tilde{y}^{k} ; \bar{a}, \bar{p}\right)$, and alternative consumption vectors $\tilde{x}^{h} \in X^{h}, h \in \mathcal{H}^{k}$, with

$$
\begin{gathered}
\sum_{h \in \mathcal{H}^{k}}\left(\tilde{p}^{\top} \tilde{x}^{h}-\bar{p}^{\top} \bar{x}^{h}\right) \leq 0, \\
\tilde{x}^{h k}=\sigma^{h k}\left(\tilde{y}^{k}\right), h \in \mathcal{H}^{k}, \\
u^{h}\left(\tilde{x}^{h}\right)>u^{h}\left(\bar{x}^{h}\right), h \in \mathcal{H}^{k} .
\end{gathered}
$$

This is the standard definition (non-existence of a superior feasible alternative), applied here to the members of union $k$ at unchanged prices for commodities not under the control of union $k\left({ }^{k} \tilde{p}={ }^{k} \bar{p}\right)$ and unchanged property incomes.

Because the perceived inverse demand functions $\pi^{k}$ are piecewise linear and the labor allocation rules are linear, we may translate that definition into an alternative formulation geared to our purpose.

Theorem 3 Under Assumptions A.6 and A.7, for given market data $(\bar{a}, \bar{p})$, for $h \in \mathcal{H}^{k}$, let $\bar{x}^{h}$ solve problem (16) with associated normalized vector of marginal utilities $\bar{\lambda}^{h}$. If

$$
\sum_{h \in \mathcal{H}^{k}}-\frac{d \sigma^{h k}}{d y^{k k}} \bar{\lambda}^{h k}-H^{k k-}(\bar{a}, \bar{p})^{\top} \bar{y}^{k k} \leq \bar{p}^{k} \leq \sum_{h \in \mathcal{H}^{k}}-\frac{d \sigma^{h k}}{d y^{k k}} \bar{\lambda}^{h k}-H^{k k+}(\bar{a}, \bar{p})^{\top} \bar{y}^{k k}
$$

where the lower bound on $\bar{p}_{l}^{k}$ is omitted for $l \in \mathcal{L}^{k}$ such that $\bar{y}_{l}^{k}=0$ and the upper bound on $\bar{p}_{l}^{k}$ is omitted for $l \in \mathcal{L}^{k}$ such that $\bar{y}_{l}^{k}=\hat{y}_{l}^{k}$, then $\left(\left(\bar{x}^{h}\right)_{h \in \mathcal{H}^{k}}, \bar{y}^{k}\right)$ is Pareto efficient for $\mathcal{H}^{k}$ at $(\bar{a}, \bar{p})$.

Proof: See Appendix.

\subsection{Pricing rules}

At a solution $k_{\bar{x}}^{h}$ to (16), it holds that the Lagrange multiplier for $h$ 's budget constraint, $\bar{\lambda}_{0}^{h}$, is equal to

$$
\max _{l \in \mathcal{L} \backslash \mathcal{L}^{k}} \frac{\partial_{x_{l}^{h}} u^{h}\left(k_{\bar{x}}^{h}, \sigma^{h k}\left(y^{k}\right)\right)}{p_{l}} .
$$

The formulation with the maximum is needed, as boundary consumption is not excluded. This motivates the definition of the function $\lambda^{h}: Y^{k} \times A \times \Delta \rightarrow \mathbb{R}^{L}$ by

$$
\lambda_{l}^{h}\left(y^{k} ; \bar{a}, \bar{p}\right)= \begin{cases}\bar{p}_{l}, & l \in \mathcal{L} \backslash \mathcal{L}^{k}, \\ \left(\min _{l^{\prime} \in \mathcal{L} \backslash \mathcal{L}^{k}} \frac{\bar{p}_{l^{\prime}}}{\partial_{x_{l^{\prime}}} u^{h}\left({ }^{k} \bar{x}^{h}, \sigma^{h k}\left(y^{k}\right)\right)}\right) \partial_{x_{l}^{h}} u^{h}\left({ }^{k} \bar{x}^{h}, \sigma^{h k}\left(y^{k}\right)\right), & l \in \mathcal{L}^{k} .\end{cases}
$$


It is not difficult to show that the function $\lambda^{h}$ is continuous. Next we define the continuous function $\lambda^{k}: Y^{k} \times A \times \Delta \rightarrow \mathbb{R}_{+}^{L}$ by

$$
\begin{aligned}
& \lambda^{k}\left(y^{k} ; \bar{a}, \bar{p}\right)={ }^{k} \bar{p} \\
& \lambda^{k k}\left(y^{k} ; \bar{a}, \bar{p}\right)=-\sum_{h \in \mathcal{H}^{k}} \frac{d \sigma^{h k}}{d y^{k k}} \lambda^{h k}\left(y^{k} ; \bar{a}, \bar{p}\right) .
\end{aligned}
$$

That is, $\lambda^{k}\left(y^{k} ; \bar{a}, \bar{p}\right)$ is a weighted average of normalized vectors of marginal utilities $\lambda^{h}\left(y^{k} ; \bar{a}, \bar{p}\right)$, with weights adding to unity. For $l \notin \mathcal{L}^{k}$, the weights are immaterial (and omitted in the above formula); for $l \in \mathcal{L}^{k}$, the weights $-\frac{d \sigma_{l}^{h k}}{d y^{k k}}$ are non-negative and add up to 1 . Thus, for $l \in \mathcal{L}^{k}, \lambda_{l}^{k}\left(y^{k} ; \bar{a}, \bar{p}\right)$ is an average reservation wage of members of $k$, weighted by marginal employment shares.

We define the pricing rule of union $k, \varphi^{k}: Y^{k} \times A \times \Delta \rightarrow \mathbb{R}^{L}$ by

$$
\begin{aligned}
\varphi^{k}\left(y^{k} ; \bar{a}, \bar{p}\right)=\left\{p^{k} \in \mathbb{R}^{L} \mid \lambda^{k}\left(y^{k} ; \bar{a}, \bar{p}\right)\right. & -H^{k-}\left(y^{k} ; \bar{a}, \bar{p}\right)^{\top} y^{k} \leq p^{k} \leq \lambda^{k}\left(y^{k} ; \bar{a}, \bar{p}\right) \\
& \left.-H^{k+}\left(y^{k} ; \bar{a}, \bar{p}\right)^{\top} y^{k}\right\},
\end{aligned}
$$

where, as before, the lower bound on $p_{l}^{k}$ is omitted for $l \in \mathcal{L}^{k}$ such that $y_{l}^{k}=0$ and the upper bound on $p_{l}^{k}$ is omitted for $l \in \mathcal{L}^{k}$ such that $y_{l}^{k}=\hat{y}_{l}^{k}$.

Observe that the pricing rule of union $k$ maps into $\mathbb{R}^{L}$ and not into $S$. Because at equilibrium $\bar{p} \in \varphi^{k}\left(y^{k} ; \bar{a}, \bar{p}\right)$ and $\bar{p} \in S$, this feature is inessential. For all $p^{k} \in \varphi^{k}\left(y^{k} ; \bar{a}, \bar{p}\right)$ we have that ${ }^{k} p^{k}={ }^{k} \bar{p}$. For $l \in \mathcal{L}^{k}$, if $p_{l}^{k}$ exceeds $\bar{p}_{l}$ for all $p^{k} \in \varphi^{k}(\bar{a}, \bar{p})$, then the union should lower $y_{l}^{k}$ at a gain of members' welfare, and the union should increase $y_{l}^{k}$ if $p_{l}^{k}$ is less than $\bar{p}_{l}$ for all $p^{k} \in \varphi^{k}\left(y^{k} ; \bar{a}, \bar{p}\right)$. Using Theorem 3 , it is immediate that if $\bar{p} \in \varphi^{k}\left(\bar{y}^{k} ; \bar{a}, \bar{p}\right)$, then $\left(\left(\bar{x}^{h}\right)_{h \in \mathcal{H}^{k}}, \bar{y}^{k}\right)$ is Pareto efficient for $\mathcal{H}^{k}$.

Instead of stating here the properties of (21) that are conducive to the existence of equilibrium, we postpone that task to Lemma 7 below, so as to encompass at once monetary exchange.

\section{The monetary economy}

Negishi (1979, p. 28) lists "monetary exchange with cash-in-advance (CIA)" as a "basic rule of the game" for macroeconomics. We now extend the model of the previous sections to introduce that rule. Yet, this (already too long) paper is not a paper on monetary theory. So, our treatment will be streamlined. It is based on Drèze and Polemarchakis (2001b), to which readers are referred for a systematic exposition and generalization.

The logic of the Drèze and Polemarchakis (2001b) model, specialized here to CIA, is elementary. All exchanges are monetary (no barter). Inside money is created at no cost by a central bank and lent by the bank to other agents against promise of repayment with interest, at rates $r$ set by the bank. Time is divided in (short) periods during which trade occurs. Money spent on purchases must be held at the beginning of a period; money collected from sales must be held idle until the end of a period. If budget constraints and profits are defined in terms of "beginning of period" values, the relevant price of 
commodity $l$ is $p_{l}$ in case of a purchase and $p_{l} /(1+r)$ in case of a sale. The bank $b$ is owned by households in given fractions $\theta^{h b} \geq 0, \sum_{h \in \mathcal{H}} \theta^{h b}=1$; bank profits (seignorage) are distributed to shareholders.

Example 4 Consider a single period example, where firm $j$ produces $y^{j}$. Define a family of diagonal $L \times L$ matrices $R\left(y^{j}\right)$ by:

$$
R_{l l}\left(y^{j}\right)= \begin{cases}1 & \text { if } y_{l}^{j}<0, \\ 1 /(1+r) & \text { if } y_{l}^{j} \geq 0 .\end{cases}
$$

The beginning-of-period value of profits is then equal to $p^{\top} R\left(y^{j}\right) y^{j}$, a piecewise linear expression with a kink when there is $l$ such that $y_{l}^{j}=0$.

In stating FOC conditions for profit maximization, one must take the kinks into account. If $y_{l}^{j}=0$, the relevant price associated with $d y_{l}^{j}>0$ is $p_{l} /(1+r)$, whereas with $d y_{l}^{j}<0$ it is $p_{l}$. This calls for defining two families of diagonal matrices, $R^{-}\left(y^{j}\right)$ and $R^{+}\left(y^{j}\right)$, where

$$
\begin{aligned}
& R_{l l}^{+}\left(y^{j}\right)= \begin{cases}1 & \text { if } y_{l}^{j}<0, \\
1 /(1+r) & \text { if } y_{l}^{j} \geq 0,\end{cases} \\
& R_{l l}^{-}\left(y^{j}\right)= \begin{cases}1 & \text { if } y_{l}^{j} \leq 0, \\
1 /(1+r) & \text { if } y_{l}^{j}>0 .\end{cases}
\end{aligned}
$$

Using these definitions, FOC like (7) and (8) may be extended to CIA, by multiplying the price-terms $p$ and $\partial p / \partial y=H$ by the suitable matrices $R^{+}$or $R^{-}$. This yields

$$
\begin{aligned}
& R^{-}\left(\bar{y}^{j}\right) p^{j} \geq q^{j}-H^{j-}(\bar{a}, \bar{p})^{\top} R^{-}\left(\bar{y}^{j}\right) \bar{y}^{j}, \\
& R^{+}\left(\bar{y}^{j}\right) p^{j} \leq q^{j}-H^{j+}(\bar{a}, \bar{p})^{\top} R^{+}\left(\bar{y}^{j}\right) \bar{y}^{j} .
\end{aligned}
$$

The pricing rules (26) below relate to (22) and (23) in basically the same way that the rules (11) relate to (7) and (8).

In a general model involving time and uncertainty, the commodity space is structured on the basis of an event tree describing the primitive uncertainties and the information of the agents. The tree has $N$ nodes, or date events, labeled $s_{t} \in \mathcal{N}$. The interest rates set by the bank are event-specific, and a distinct CIA constraint applies at each date event $s_{t}$. The Drèze and Polemarchakis (2001b) paper explains how the corresponding detailed model can be consolidated into an abstract representation in terms of present value prices for contingent commodities; that construction extends chapter 6 of Debreu (1959) to a streamlined monetary economy. The consolidation is particularly straightforward under the (special) CIA transactions technology. We refer readers to Drèze and Polemarchakis for details and introduce directly the consolidated version of the model. Under CIA, it is not even necessary to bring out into the open holdings of money or nominal assets; so long as the bank supplies cash as demanded by solvent agents, the only relevant consideration is the discounting of sales receipts under date event $s_{t}$ by the discount factor $1 /\left(1+r_{s_{t}}\right)$ applicable there; that discount factor applies unchanged to present value prices, which 
are based on "beginning of period accounting" at each date event. The only additional complication concerns the seignorage revenue of the bank. In a streamlined consolidation where nominal assets remain implicit, and CIA applies, the bank is assumed to collect interest for one period on all the cash used by the agents for their purchases at a date event. In the aggregate, that amount is identical to terminal cash holdings of the sellers. The bank revenues (seignorage) are distributed at the end of the period to households as dividends.

Formally, the commodity space is now $\mathbb{R}^{L N}$ and the vector $p \in \mathbb{R}_{+}^{L N}$ denotes present values. We write $p^{\top}=\left(p_{s_{t}}^{\top}\right)_{s_{t} \in \mathcal{N}}$, where $p_{s_{t}} \in \mathbb{R}_{+}^{L}$ is the vector of present values (at node, or time 0 ) of commodities purchased contingently on date event $s_{t}$. For sales of commodity $l$ at $s_{t}$, the relevant price is $p_{l s_{t}} /\left(1+r_{s_{t}}\right)$, where $r_{s_{t}} \geq 0$ is part of the primitives (is exogenously set by the bank).

In line with Example 4, we define pairs of diagonal matrices $R^{+}\left(y^{j}\right), R^{-}\left(y^{j}\right)$ of dimension $L N$ by

$$
\begin{aligned}
& R_{l s_{t}, l_{t}}^{+}\left(y^{j}\right)= \begin{cases}1 & \text { if } y_{l s_{t}}^{j}<0, \\
1 /\left(1+r_{s_{t}}\right) & \text { if } y_{l_{s_{t}}}^{j} \geq 0,\end{cases} \\
& R_{l_{s_{t}}, l_{s_{t}}}^{-}\left(y^{j}\right)= \begin{cases}1 & \text { if } y_{l_{s_{t}}}^{j} \leq 0, \\
1 /\left(1+r_{s_{t}}\right) & \text { if } y_{l_{s_{t}}}^{j}>0 .\end{cases}
\end{aligned}
$$

The present value of profits of firm $j$ is then: ${ }^{26}$

$$
\hat{v}^{j}=p^{\top} R^{+}\left(y^{j}\right) y^{j}\left(=p^{\top} R^{-}\left(y^{j}\right) y^{j}\right) .
$$

Similarly, denoting by $z^{h}:=x^{h}-e^{h}$ the transactions of household $h$, we define diagonal matrices $R^{\circ}\left(z^{h}\right)$ by:

$$
R_{l s_{t}, l s_{t}}^{\circ}\left(z^{h}\right)= \begin{cases}1 /\left(1+r_{s_{t}}\right) & \text { if } z_{l s_{t}}^{h} \leq 0, \\ 1 & \text { if } z_{l_{s_{t}}}^{h}>0 .\end{cases}
$$

We denote by $\hat{v}^{h}$ the dividend income of household $h, \hat{v}^{h}=\sum_{j \in \mathcal{J}} \theta^{h j} \hat{v}^{j}+\theta^{h b} \hat{v}^{b}$, where $\hat{v}^{b}$ represents the present value of seignorage. It holds that

$$
\hat{v}^{b}=\sum_{s_{t} \in \mathcal{N}} \frac{r_{s_{t}}}{1+r_{s_{t}}}\left(\sum_{j \in \mathcal{J}} p_{s_{t}}^{\top} y_{-s_{t}}^{j}+\sum_{h \in \mathcal{H}} p_{s_{t}}^{\top} z_{+s_{t}}^{h}\right),
$$

where we use the notation, for $v \in \mathbb{R}^{n}, v_{+i}:=\max \left(0, v_{i}\right)$ and $v_{-i}:=\max \left(0,-v_{i}\right)$, so that $v=v_{+}-v_{-}$.

The consolidated budget constraint of $h$ is

$$
p^{\top} R^{\circ}\left(z^{h}\right) z^{h} \leq \hat{v}^{h} .
$$

Pricing rules in the monetary economy are natural extensions of earlier sections.

\footnotetext{
${ }^{26}$ For profit calculations given $y^{j}$, the distinction between $R^{+}\left(y^{j}\right)$ and $R^{-}\left(y^{j}\right)$ is unnecessary; for defining pricing rules, the distinction is necessary, as illustrated in Example 4.
} 
In the monetary economy with linear perceived inverse demand functions, the firm's problem is:

$$
\max _{y^{j} \in Y^{j}} y^{j^{\top}} R^{+}\left(y^{j}\right) \pi^{j}\left(y^{j} ; \bar{a}, \bar{p}\right)=y^{j^{\top}} R^{+}\left(y^{j}\right)\left(\bar{p}-H^{j}(\bar{a}, \bar{p}) \bar{y}^{j}\right)+y^{j^{\top}} R^{+}\left(y^{j}\right) H^{j}(\bar{a}, \bar{p}) y^{j} .
$$

In the more general specification of Section 3, with a kinky perceived inverse demand function, we can replace the sufficient conditions (7) and (8) by the sufficient conditions

$$
\begin{aligned}
& \bar{p} \geq R^{-}\left(\bar{y}^{j}\right)^{-1} q^{j}-R^{-}\left(\bar{y}^{j}\right)^{-1} H^{j-}(\bar{a}, \bar{p})^{\top} R^{-}\left(\bar{y}^{j}\right) \bar{y}^{j}, \\
& \bar{p} \leq R^{+}\left(\bar{y}^{j}\right)^{-1} q^{j}-R^{+}\left(\bar{y}^{j}\right)^{-1} H^{j+}(\bar{a}, \bar{p})^{\top} R^{+}\left(\bar{y}^{j}\right) \bar{y}^{j} .
\end{aligned}
$$

To prove existence, we must extend Assumptions A. $3^{\prime}$ and A.5 to

(A.3") For each $j \in \mathcal{J}$, the mapping $\left(H^{j-}, H^{j+}\right): \partial Y^{j} \times A \times \Delta^{L N} \rightarrow \mathbb{R}^{2 L^{2} N^{2}}$, which maps $\left(y^{j}, \bar{a}, \bar{p}\right)$ to $\left(H^{j-}\left(y^{j} ; \bar{a}, \bar{p}\right), H^{j+}\left(y^{j} ; \bar{a}, \bar{p}\right)\right)$, is continuous and the matrices $R^{-}\left(y^{j}\right) H^{j-}\left(y^{j} ; \bar{a}, \bar{p}\right), \quad R^{+}\left(y^{j}\right) H^{j+}\left(y^{j} ; \bar{a}, \bar{p}\right)$, as well as the derived matrices $R^{+}\left(y^{j}\right) H^{j s}\left(y^{j} ; \bar{a}, \bar{p}\right), \quad s \in \mathcal{S}$, are NSD. It holds that $\left(H^{j-}\left(\bar{y}^{j} ; \bar{a}, \bar{p}\right), H^{j+}\left(\bar{y}^{j} ; \bar{a}, \bar{p}\right)\right)=\left(H^{j-}(\bar{a}, \bar{p}), H^{j+}(\bar{a}, \bar{p})\right)$.

(A.5') For each $j \in \mathcal{J}$ and for each $y^{j} \in \partial Y^{j}$, the inequalities

$$
R^{+}\left(y^{j}\right)^{-1} H^{j+}\left(y^{j} ; \bar{a}, \bar{p}\right)^{\top} R^{+}\left(y^{j}\right) y^{j} \leq R^{-}\left(y^{j}\right)^{-1} H^{j-}\left(y^{j} ; \bar{a}, \bar{p}\right)^{\top} R^{-}\left(y^{j}\right) y^{j}
$$

hold.

We define

$M^{j}\left(y^{j} ; \bar{a}, \bar{p}\right)=\frac{1}{2} R^{-}\left(y^{j}\right)^{-1} H^{j-}\left(y^{j} ; \bar{a}, \bar{p}\right)^{\top} R^{-}\left(y^{j}\right)+\frac{1}{2} R^{+}\left(y^{j}\right)^{-1} H^{j+}\left(y^{j} ; \bar{a}, \bar{p}\right)^{\top} R^{+}\left(y^{j}\right)$

and

$$
\mu^{j}\left(y^{j} ; \bar{a}, \bar{p}\right)=-\iota^{\top} M^{j}\left(y^{j} ; \bar{a}, \bar{p}\right) y^{j} .
$$

We define pricing rules for firms in a monetary economy with kinky perceived inverse demands as follows:

$$
\begin{aligned}
& \varphi^{j}\left(y^{j} ; \bar{a}, \bar{p}\right)=\left\{p^{j} \in S^{L N} \mid \exists \bar{q}^{j} \in N^{j}\left(y^{j}\right) \text { with } \iota^{\top} R^{+}\left(y^{j}\right)^{-1} \bar{q}^{j}=1\right. \text { such that } \\
& R^{-}\left(y^{j}\right)^{-1} \bar{q}^{j} \max \left(0,1-\mu^{j}\left(y^{j} ; \bar{a}, \bar{p}\right)\right)-\frac{R^{-}\left(y^{j}\right)^{-1} H^{j-}\left(y^{j} ; \bar{a}, \bar{p}\right)^{\top} R^{-}\left(y^{j}\right) y^{j}}{\max \left(1, \mu^{j}\left(y^{j} ; \bar{a}, \bar{p}\right)\right)} \leq p^{j} \leq \\
& \left.R^{+}\left(y^{j}\right)^{-1} \bar{q}^{j} \max \left(0,1-\mu^{j}\left(y^{j} ; \bar{a}, \bar{p}\right)\right)-\frac{R^{+}\left(y^{j}\right)^{-1} H^{j+}\left(y^{j} ; \bar{a}, \bar{p}\right)^{\top} R^{+}\left(y^{j}\right) y^{j}}{\max \left(1, \mu^{j}\left(y^{j} ; \bar{a}, \bar{p}\right)\right)}\right\} .
\end{aligned}
$$

We now state Lemma 6.

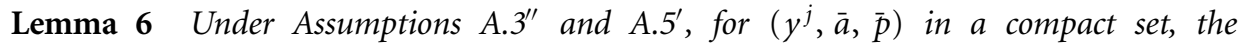
pricing rule (26) satisfies $P .1$ with $\iota^{\top} p^{j} \equiv 1$ for every $p^{j} \in \varphi^{j}\left(y^{j} ; \bar{a}, \bar{p}\right)$. For all $p^{j} \in \varphi^{j}\left(y^{j} ; \bar{a}, \bar{p}\right), p^{j^{\top}} R^{+}\left(y^{j}\right) y^{j} \geq 0$. 
Proof: See Appendix.

We now extend the definition of vectors $\left(\left(\bar{x}^{h}\right)_{h \in \mathcal{H}^{k}}, \bar{y}^{k}\right)$ that are Pareto efficient from the viewpoint of union members to the monetary case.

Definition 4 Consumption bundles and employment levels $\left(\left(\bar{x}^{h}\right)_{h \in \mathcal{H}^{k}}, \bar{y}^{k}\right)$ with associated prices (i.e. wages) $\pi^{k}\left(\bar{y}^{k} ; \bar{a}, \bar{p}\right)$ are Pareto efficient for $\mathcal{H}^{k}$ at market signals $(\bar{a}, \bar{p})$ if there do not exist alternative employment levels $\tilde{y}^{k} \in Y^{k}$ with associated prices $\tilde{p}=\pi^{k}\left(\tilde{y}^{k} ; \bar{a}, \bar{p}\right)$, and alternative consumption vectors $\tilde{x}^{h} \in X^{h}, h \in \mathcal{H}^{k}$, with

$$
\begin{gathered}
\sum_{h \in \mathcal{H}^{k}}\left(\tilde{p}^{\top} R^{\circ}\left(\tilde{x}^{h}-e^{h}\right) \tilde{x}^{h}-\bar{p}^{\top} R^{\circ}\left(\bar{x}^{h}-e^{h}\right) \bar{x}^{h}\right) \leq 0, \\
\tilde{x}^{h k}=\sigma^{h k}\left(\tilde{y}^{k}\right), h \in \mathcal{H}^{k}, \\
u^{h}\left(\tilde{x}^{h}\right)>u^{h}\left(\bar{x}^{h}\right), h \in \mathcal{H}^{k} .
\end{gathered}
$$

This is the standard definition (non-existence of a superior feasible alternative), applied here to the members of union $k$ at unchanged prices for commodities not under the control of union $k\left({ }^{k} \tilde{p}={ }^{k} \bar{p}\right)$ and unchanged property incomes.

We extend Assumption A.7 to

$($ A.7' $)$ For each $k \in \mathcal{K}$, the mapping $\left(H^{k-}, H^{k+}\right): Y^{k} \times A \times \Delta^{L N} \rightarrow \mathbb{R}^{2 L^{2} N^{2}}$, which maps $\left(y^{k} ; \bar{a}, \bar{p}\right)$ to $\left(H^{k-}\left(y^{k} ; \bar{a}, \bar{p}\right), H^{k+}\left(y^{k} ; \bar{a}, \bar{p}\right)\right)$, is continuous and the matrices

$$
R^{+}\left(y^{k}\right) H^{k-}\left(y^{k} ; \bar{a}, \bar{p}\right), R^{+}\left(y^{k}\right) H^{k+}\left(y^{k} ; \bar{a}, \bar{p}\right),
$$

as well as the derived matrices $R^{+}\left(y^{k}\right) H^{k s}\left(y^{k} ; \bar{a}, \bar{p}\right), s \in \mathcal{S}$, are NSD. It holds that $\left(H^{k-}\left(\bar{y}^{k} ; \bar{a}, \bar{p}\right), H^{k+}\left(\bar{y}^{k} ; \bar{a}, \bar{p}\right)\right)=\left(H^{k-}(\bar{a}, \bar{p}), H^{k+}(\bar{a}, \bar{p})\right)$. For each $k \in \mathcal{K}$ and for each $y^{k} \in Y^{k}$, the matrices $H^{k-}\left(y^{k} ; \bar{a}, \bar{p}\right)$ and $H^{k+}\left(y^{k} ; \bar{a}, \bar{p}\right)$ have rows and columns of zeros for all commodities $l s_{t} \in(\mathcal{L} \times \mathcal{N}) \backslash\left(\mathcal{L}^{k} \times \mathcal{N}\right)$, and the inequalities

$$
H^{k+}\left(y^{k} ; \bar{a}, \bar{p}\right)^{\top} R^{+}\left(y^{k}\right) y^{k} \leq H^{k-}\left(y^{k} ; \bar{a}, \bar{p}\right)^{\top} R^{+}\left(y^{k}\right) y^{k}
$$

hold.

Household $h$ solves the problem

$$
\begin{aligned}
\max _{{ }^{k} x^{h}} u^{h}\left({ }^{k} x^{h}, \sigma^{h k}\left(y^{k}\right)\right) \text { s.t. } & p^{\top} R^{\circ}\left({ }^{k} x^{h}-{ }^{k} e^{h}, \sigma^{h k}\left(y^{k}\right)\right)\left({ }^{k} x^{h}-{ }^{k} e^{h}, \sigma^{h k}\left(y^{k}\right)\right) \leq \hat{v}^{h}, \\
& \left({ }^{k} x^{h}, \sigma^{h k}\left(y^{k}\right)\right) \in X^{h} .
\end{aligned}
$$

Associated with a solution $\bar{x}^{h}=\left({ }^{k} \bar{x}^{h}, \sigma^{h k}\left(y^{k}\right)\right)$ of problem (30), we define the normalized vector of marginal utilities $\bar{\lambda}^{h}$ by $\bar{\lambda}_{l s_{t}}^{h}=p_{l s_{t}}, \quad l s_{t} \in(\mathcal{L} \times \mathcal{N}) \backslash\left(\mathcal{L}^{k} \times \mathcal{N}\right)$, and $\bar{\lambda}_{l s_{t}}^{h}=\partial_{x_{l s_{t}}^{h}} u^{h}\left(\bar{x}^{h}\right) / \bar{\lambda}_{0}^{h}, l s_{t} \in \mathcal{L}^{k} \times \mathcal{N}$, where $\bar{\lambda}_{0}^{h}$ denotes the Lagrange multiplier for $h$ 's budget constraint. Assumption A.6.2 implies that $\bar{\lambda}_{0}^{h}>0$.

As before, we may now characterize the vectors $\left(\left(\bar{x}^{h}\right)_{h \in \mathcal{H}^{k}}, \bar{y}^{k}\right)$ that are Pareto efficient from the viewpoint of union members. The proof is an extension of the proof of Theorem 3 and is omitted here. 
Theorem 4 Under Assumptions A.6 and A.7', for given market data $(\bar{a}, \bar{p})$, for $h \in \mathcal{H}^{k}$, let $\bar{x}^{h}$ solve problem (30) with associated normalized vector of marginal utilities $\bar{\lambda}^{h}$. If

$$
\begin{aligned}
& \sum_{h \in \mathcal{H}^{k}}-R^{k+}\left(\bar{y}^{k}\right)^{-1} \frac{d \sigma^{h k}}{d y^{k k}} \bar{\lambda}^{h k}-H^{k k-}(\bar{a}, \bar{p})^{\top} \bar{y}^{k k} \leq \bar{p}^{k} \\
& \leq \sum_{h \in \mathcal{H}^{k}}-R^{k+}\left(\bar{y}^{k}\right)^{-1} \frac{d \sigma^{h k}}{d y^{k k}} \bar{\lambda}^{h k}-H^{k k+}(\bar{a}, \bar{p})^{\top} \bar{y}^{k k},
\end{aligned}
$$

where the lower bound on $\bar{p}_{l_{s_{t}}}^{k}$ is omitted for $l_{s_{t}} \in \mathcal{L}^{k} \times \mathcal{N}$ such that $\bar{y}_{l s_{t}}^{k}=0$ and the upper

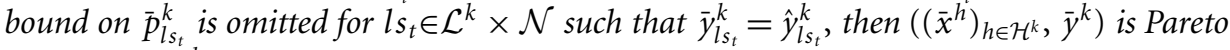
efficient for $\mathcal{H}^{k}$ at $(\bar{a}, \bar{p})$.

In Theorem 4 we have written $R^{k+}\left(\bar{y}^{k}\right)$ for the $L^{k} N \times L^{k} N$ submatrix of $R^{+}\left(\bar{y}^{k}\right)$ corresponding to the rows and columns in $\mathcal{L}^{k} \times \mathcal{N}$.

At a solution ${ }^{k} \bar{x}^{h}$ to (30) with $\hat{v}^{h} \geq 0$, it holds that the Lagrange multiplier for $h$ 's budget constraint, $\bar{\lambda}_{0}^{h}$, is equal to

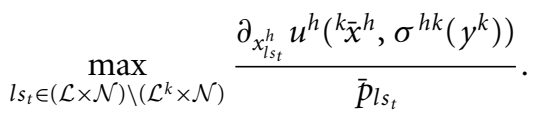

Therefore, we can define the functions $\lambda^{h}$ and $\lambda^{k}$ as in the case without money and we define the pricing rule of union $k, \varphi^{k}: Y^{k} \times A \times \Delta^{L N} \rightarrow \mathbb{R}^{L N}$ by

$$
\begin{aligned}
& \varphi^{k}\left(y^{k} ; \bar{a}, \bar{p}\right)=\left\{p^{k} \in \mathbb{R}^{L N} \mid R^{+}\left(y^{k}\right)^{-1} \lambda^{k}\left(y^{k} ; \bar{a}, \bar{p}\right)-H^{k-}\left(y^{k} ; \bar{a}, \bar{p}\right)^{\top} y^{k} \leq p^{k}\right. \\
& \left.\quad \leq R^{+}\left(y^{k}\right)^{-1} \lambda^{k}\left(y^{k} ; \bar{a}, \bar{p}\right)-H^{k+}\left(y^{k} ; \bar{a}, \bar{p}\right)^{\top} y^{k}\right\},
\end{aligned}
$$

where, as before, the lower bound on $p_{l s_{t}}^{k}$ is omitted for $l s_{t} \in \mathcal{L}^{k} \times \mathcal{N}$ such that $y_{l s_{t}}^{k}=0$ and the upper bound on $p_{l s_{t}}^{k}$ is omitted for $l s_{t} \in \mathcal{L}^{k} \times \mathcal{N}$ such that $y_{l s_{t}}^{k}=\hat{y}_{l s_{t}}^{k}$.

Due to the omission of lower and upper bounds when $y_{l s_{t}}^{k}=0$ and $y_{l s_{t}}^{k}=\hat{y}_{l s_{t}}^{k}$, the correspondence $\varphi^{k}$ has unbounded sets as images. For $k \in \mathcal{K}$, let $\tilde{\varphi}^{k}\left(y^{k} ; a, p\right)$ be defined as the subset of $\varphi^{k}\left(y^{k} ; a, p\right)$ as defined in (32) that satisfies the lower bound on $p_{l s_{t}}^{k}$ for $l s_{t} \in\left(\mathcal{L}^{k} \times \mathcal{N}\right)$ such that $y_{l s_{t}}^{k}=0$ and the upper bound on $p_{l s_{t}}^{k}$ for $l s_{t} \in \mathcal{L}^{k} \times \mathcal{N}$ such that $y_{l s_{t}}^{k}=\hat{y}_{l s_{t}}^{k}$. We will make use of $\tilde{\varphi}^{k}$ in the equilibrium existence proof.

Lemma 7 Under Assumptions (A.3' $),\left(A .5^{\prime}\right),(A .6)$ and $\left(A .7^{\prime}\right)$, for $\left(y^{k}, \bar{a}, \bar{p}\right) \in Y^{k} \times A \times$ $\Delta^{L N}$, the correspondence $\tilde{\varphi}^{k}$ satisfies P.1.

Proof: The proof follows easily using the continuity of the function $\lambda^{k}$.

Lemmas 6 and 7 are crucial to our main result, the existence theorem in Section 6.

\section{Existence of Keynes-Negishi equilibria}

We may now bring together the contents of Sections 2-5. The primitives of the economy $\mathcal{E}$ are given by (a)-(d). Beyond the properties of the commodity space in (a), $Y^{j}$ in (b), $\succeq_{h}$ in 
(c) and the union in (d), the assumptions are: A.1 and A.2 in Section 2, A.4' in Section 3, A.6 in Section 4, and A.3", A. $5^{\prime}$ and A.7' in Section 5.

Definition 5 A Keynes-Negishi equilibrium for $\mathcal{E}$ is a tuple $\left(p \in \Delta^{L N}, r \in \mathbb{R}_{+}^{N}\right.$,

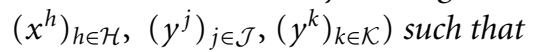

(i) for each $h \in \mathcal{H}, x^{h}$ is $\succeq_{h}$-maximal over the set

$$
\begin{aligned}
\left\{\tilde{x}^{h} \in X^{h} \mid\right. & p^{\top} R^{\circ}\left(\tilde{z}^{h}\right) \tilde{z}^{h} \leq \hat{v}^{h} ; \\
& \text { if } \left.h \in \mathcal{H}^{k}, \tilde{x}_{l s_{t}}^{h}=\sigma_{l s_{t}}^{h k} y_{l_{s_{t}}}^{k} \quad \text { for all } l s_{t} \in \mathcal{L}^{k} \times \mathcal{N}\right\},
\end{aligned}
$$

where, for $j \in \mathcal{J}, \quad \hat{v}^{j}=p^{\top} R^{+}\left(y^{j}\right) y^{j}, \quad \hat{v}^{b}=\sum_{h \in \mathcal{H}} p^{\top}\left(I-R^{\circ}\left(z^{h}\right)\right) z^{h}+\sum_{j \in \mathcal{J}}$ $p^{\top}\left(I-R^{+}\left(y^{j}\right)\right) y^{j}$, and $\hat{v}^{h}=\sum_{j \in \mathcal{J}} \theta^{h j} \hat{v}^{j}+\theta^{h b} \hat{v}^{b}$

(ii) for each $j \in \mathcal{J}, y^{j} \in Y^{j}$; for all $\tilde{y}^{j} \in Y^{j}, \tilde{y}^{j} \in \mathbb{R}^{L N}\left(y^{j}, s\right)$ for some sign vector $s, p^{\top} R^{+}\left(y^{j}\right) y^{j} \geq\left(p+H^{j s}(a, p)\left(\tilde{y}^{j}-y^{j}\right)\right)^{\top} R^{+}\left(\tilde{y}^{j}\right) \tilde{y}^{j}$

(iii) for each $k \in \mathcal{K}, y^{k} \in Y^{k}$; and $\left(\left(x^{h}\right)_{h \in \mathcal{H}^{k}}, y^{k}\right)$ is Pareto efficient for $\mathcal{H}^{k}$ at $(a, p)$, so there do not exist $\tilde{y}^{k} \in Y^{k}, \tilde{y}^{k} \in \mathbb{R}^{L N}\left(y^{k}, s\right)$ for some sign vector $s$, and, for $h \in \mathcal{H}^{k}, \tilde{x}^{h} \in X^{h}$, where $\tilde{p}=H^{k s}(a, p)\left(\tilde{y}^{k}-y^{k}\right)+p$ such that

$$
\begin{aligned}
& \sum_{h \in \mathcal{H}^{k}}\left(\tilde{p}^{\top} R^{\circ}\left(\tilde{z}^{h}\right) \tilde{x}^{h}-p^{\top} R^{\circ}\left(z^{h}\right) x^{h}\right) \leq 0, \\
& \tilde{x}^{h k}=\sigma^{h k}\left(\tilde{y}^{k}\right), h \in \mathcal{H}^{k}, \\
& \tilde{x}^{h} \succ^{h} x^{h}, h \in \mathcal{H}^{k} ;
\end{aligned}
$$

(iv) $\sum_{h \in \mathcal{H}} x^{h}=\sum_{h \in \mathcal{H}} e^{h}+\sum_{j \in \mathcal{J}} y^{j}$.

The unfamiliar conditions in (ii) and (iii) admit a natural interpretation. The conditions in (ii) state that profits at $y^{j}$ computed at equilibrium prices $(p, r)$ are at least as high as profits at any feasible alternative $\tilde{y}^{j}$, taking into account the price adjustments $H^{j s}(\cdot)\left(\tilde{y}^{j}-y^{j}\right)$ associated with the move from $y^{j}$ to $\tilde{y}^{j}$. These adjustments bring in the matrix of price derivatives $H^{j s}(\cdot)$ geared to the signs $s_{l_{s_{t}}}$ of the quantity adjustments $\left(\tilde{y}_{l s_{t}}^{j}-y_{l_{s_{t}}}^{j}\right)$. Similarly, the conditions in (iii) state that $\left(\left(x^{h}\right)_{h \in \mathcal{H}^{k}}, y^{k}\right)$ cannot be Pareto improved by an admissible tuple $\left(\left(\tilde{x}^{h}\right)_{h \in \mathcal{H}^{k}}, \tilde{y}^{k}\right)$ taking into account the price adjustments $H^{k s}(\cdot)\left(\tilde{y}^{k}-y^{k}\right)$ associated with the move from $y^{k}$ to $\tilde{y}^{k}$. These adjustments bring in the matrix of price derivatives $H^{k s}(\cdot)$ geared to the signs $s_{l_{s_{t}}}$ of the quantity adjustments $\left(\tilde{y}_{l_{s_{t}}}^{k}-y_{l_{s_{t}}}^{k}\right)$. Conditions (ii) and (iii) may be written alternatively as:

(ii') for each $j \in \mathcal{J}, y^{j} \in Y^{j}$ and $p \in \varphi^{j}\left(y^{j} ; a, p\right)$ as defined by (26);

(iii') for each $k \in \mathcal{K}, y^{k} \in Y^{k}$ and $p \in \varphi^{k}\left(y^{k} ; a, p\right)$ as defined by (32).

It is readily verified that a tuple $\left(p \in \mathbb{R}_{+}^{L N}, \quad r \in \mathbb{R}_{+}^{N}, \quad\left(x^{h}\right)_{h \in \mathcal{H}}, \quad\left(y^{j}\right)_{j \in \mathcal{J}},\left(y^{k}\right)_{k \in \mathcal{K}}\right)$ satisfying conditions (i) and (iv) of Definition 5, and additionally the requirement that for $j \in \mathcal{J}, \quad p \in \varphi^{j}\left(y^{j} ; a, p\right)$, and for $k \in \mathcal{K}, \quad p \in \varphi^{k}\left(y^{k} ; a, p\right)$, is a Keynes-Negishi equilibrium.

Theorem 5 For the economy $\mathcal{E}$ defined by $(a)-(d)$, under assumptions A.1, A.2, A.3", A.4', A. $5^{\prime}$, A. 6 and A. $7^{\prime}$, given any $r \in \mathbb{R}_{+}^{N}$, there exists a Keynes-Negishi equilibrium. 
Proof: See Appendix.

Until now, prices have been normalized to belong to $\Delta^{L N}$. In a monetary economy, it is not natural to do so. It is straightforward to extend all the previous definitions and results to the case where $p \in \mathbb{R}_{+}^{L N} \backslash\{0\}$. We make the following homogeneity assumption.

(A.8) For $j \in \mathcal{J}$, for $k \in \mathcal{K}$, for $(\bar{a}, \bar{p}) \in A \times \Delta^{L N}$, we assume that for all $\gamma>0$,

$$
\begin{array}{ll}
\pi^{j}\left(y^{j} ; \bar{a}, \gamma \bar{p}\right)=\gamma \bar{p}+\gamma H^{j s}(\bar{a}, \bar{p})\left(y^{j}-\bar{y}^{j}\right), & s \in \mathcal{S}, y^{j} \in \mathbb{R}^{L N}\left(\bar{y}^{j}, s\right), \\
\pi^{k}\left(y^{k} ; \bar{a}, \gamma \bar{p}\right)=\gamma \bar{p}+\gamma H^{k s}(\bar{a}, \bar{p})\left(y^{k}-\bar{y}^{k}\right), & s \in \mathcal{S}, y^{k} \in \mathbb{R}^{L N}\left(\bar{y}^{k}, s\right) .
\end{array}
$$

Now we have the following result.

Theorem 6 For the economy $\mathcal{E}$ defined by (a)-(d), under assumptions A.1, A.2, A.3", A.4', A.5', A.6, A.7' and A.8, given any $r \in \mathbb{R}_{+}^{N}$, there exists a Keynes-Negishi equilibrium. Moreover, if $\left(p, r,\left(x^{h}\right)_{h \in \mathcal{H}},\left(y^{j}\right)_{j \in \mathcal{J}},\left(y^{k}\right)_{k \in \mathcal{K}}\right)$ is a Keynes-Negishi equilibrium, then so is $\left(\gamma p, r,\left(x^{h}\right)_{h \in \mathcal{H}},\left(y^{j}\right)_{j \in \mathcal{J}},\left(y^{k}\right)_{k \in \mathcal{K}}\right)$ for any $\gamma>0$.

Proof: See Appendix.

Remark 1 Theorem 6 establishes existence of equilibria, indeterminacy of the overall price level, and neutrality of the overall price level, because the real part of equilibria is unaffected by the overall price level. We return to the interpretation of this dichotomy in Section 7.2.2. However, we must report here and now that Drèze and Polemarchakis (2001b) (Proposition 2, p. 104) prove a much stronger indeterminacy result: "Let $\mathcal{S}_{t}$, the set of possible date events at time $t$, contain $N_{t}$ elements; for $\left(c_{1}, \ldots, c_{N_{t}}\right) \in \mathbb{R}_{++}^{N_{t}}$, otherwise arbitrary, there exists a Keynes-Negishi equilibrium with $\sum_{l} \tilde{p}_{l s_{t}}=c_{s_{t}}$, all $s_{t} \in \mathcal{S}_{t}$." The meaning of this result is that not only the overall price level but also the variability of inflation rates are indeterminate; and today's price level cannot be inferred from yesterday's price level and rate of interest. Subject to formal verification, the same property should hold in the present model.

\section{Discussion}

\subsection{Assessment}

Theorem 6 accomplishes precisely the purpose stated at the outset of Section 1.2. Good. That carries two implications: (i) it shows that Negishi's approach to Keynesian economics is coherent, not only in aggregated yet simplified models amenable to explicit solutions, but also in models and under assumptions of substantial generality; (ii) it shows how kinky perceived demands can be fitted into general equilibrium theory (GET), thereby opening the way to incorporation of that feature into more sophisticated and more realistic models: like general equilibrium with incomplete markets or temporary general equilibrium (TGE).

Ad (ii), it should be noted that more sophisticated models are needed to account endogenously for two features taken for granted in the present paper, namely imperfect competition on both product and labor markets. Examples of primitive sources of imperfect 
competition include: for product markets, the presence of fixed costs or increasing returns to scale (see Dehez et al. 2003); and for labor markets, the role of wage rigidities in improving risk sharing under incomplete markets (see Drèze and Gollier 1993; Herings and Polemarchakis 2005). GET with such features is possible, but demanding.

But how far does Theorem 6 take us along the program of Negishi's book entitled Microeconomic Foundations of Keynesian Macroeconomics? In particular, does this paper conform to the methodological options advocated by Negishi? Does it lead to applications (meaning macroeconomic applications), as advocated in the citation up front?

\subsection{Methodology}

Regarding methodology, three remarks are in order.

\subsubsection{Money}

Negishi (1979) insists that the economics of Walras are unsuited to provide foundations for a theory of prolonged unemployment, because: (i) Walras proceeds stepwise from a real equilibrium, supposedly attained through tatonnement, to the same equilibrium with monetary exchange, thus assigning to money an ancillary role relative to real equilibrium; and (ii) price setting agents are needed to make sense of the price rigidities causing prolonged unemployment. ${ }^{27}$

The present paper also proceeds stepwise from a real equilibrium to a monetary equilibrium. It should, however, be clear from Theorem 6 that the order of presentation, guided by pedagogical considerations, is immaterial: the theorem brings the real and monetary aspects together (without "order") into an integrated model, for which existence of equilibrium is proved. However, an existence theorem is silent about the process through which equilibrium is reached. Existence theory alone does not preempt a particular adjustment process. Such is the case for any equilibrium concept, whether, for instance, competitive or Keynesian.

\subsubsection{Short-run dynamics}

The fact that the overall price level is indeterminate and neutral under Theorem 6 does not result from the order of presentation, but from the modeling assumptions for the monetary economy, in particular the absence of initial nominal positions or constraints. ${ }^{28}$ And yet, there is no doubt that the main determinant of today's price level is yesterday's price level!

To generate realistic substantive implications, monetary theory must encompass the dynamics of nominal price formation. In particular, nominal stickiness is required to allow nominal interest rates set by monetary authorities to affect real rates perceived by the agents. The fact that a model of transaction demand for money (like CIA) leaves both the overall price level and the variability (across successor date events) of inflation rates undetermined

\footnotetext{
${ }^{27}$ See Negishi (1979).

${ }^{28}$ We note in passing that the levels of nominal interest rates are not neutral; see Corollary 3 in Drèze and Polemarchakis (2001b).
} 
is ultimately a saving grace: it leaves room for the specification, and working, of short-run nominal dynamics! But these are not part of the existence theory presented here.

One of the merits of introducing kinky demand perceptions is definitely to open the way to interesting short-run nominal dynamics with price stickiness: interesting, because the price stickiness is not imposed from outside but rooted in natural information asymmetries; interesting also because the price dynamics under kinky demands could be varied. Whether or not the location of the kink adjusts to input prices, for instance, can make a lot of difference!

\subsubsection{Pricing rules}

Pricing rules may seem like a promising concept for modeling nominal price formation. For instance, it was noted above that a competitive firm might be modeled as setting prices equal to marginal costs, thus bypassing the difficulty raised in Arrow (1959). Yet, it should be recognized that the pricing rules introduced above are first and foremost a tool of the economist eager to verify the "consistency of a theoretical structure" through an existence theorem. The actual price-setting behavior of a firm holding asymmetrical perceptions of its demand elasticities is apt to be more streamlined than suggested by formula (38); this is especially the case for unions! But it is also apt to be more complex: for instance reflecting costs of changing prices or adoption of a periodical pattern of staggered price adjustments. Once again, there is a long way from existence theorems to the dynamics of short-run adjustment.

\subsection{Applications}

The main application pursued in Negishi (1979) concerns the role of aggregate demand in determining the levels of output and employment. Under a sufficiently widespread degree of price rigidity due to kinky demand perceptions, the economy should react to shocks in aggregate demand through quantity adjustments at unchanged prices. Attributing such shocks to whimsical shifts in expectations affecting investment demand but not savings delivers the Keynesian conclusions.

The framework of the present paper is the abstract and essentially static "complete markets" model of GET, incorporating Walras law and Say's law. Therefore, it does not assign a role to "aggregate demand"; and it is ill-suited to study "whimsical shifts in expectations". The more realistic TGE model would be better suited for that purpose. As noted under (ii) in Section 7.1, one potential byproduct of this paper is to open the way towards analysis of TGE models with kinky perceived demand.

Still, fitting shifts in expectations into the GET model is possible, if the event tree is expanded to incorporate unobserved events affecting the expectations of (some) agents, hence their market demands. (In an expected utility formulation, the shifts in expectations operate on subjective probabilities, at unchanged consumption preferences or production possibilities given date events.)

The question then arises: can one establish, for our general model, that expectationsinduced changes in aggregate (investment) demand result in quantity adjustments at unchanged prices, at least within a range? In comparison with the simple aggregated models used in macroeconomics, and in Negishi (1979), our model is more versatile, which is both 
a drawback and a merit. In particular, we have allowed for an arbitrary mix of perfectly or imperfectly competitive firms and labor markets; also for an arbitrary mix of kinky or smooth perceived demands. Therefore, we would expect mixed reactions in terms of prices and quantities.

A suggestive analogy comes from the study of economies where some prices are fixed (group I) and others are flexible (group II); see, in particular, Herings (1996), Drèze (1997) and Citanna et al. (2001) or Drèze (2001) for a non-technical summary. The main result from that literature is this: given arbitrarily fixed group I prices, there exists a continuum of supply-contrained equilibria (i.e. equilibria with quantity constraints on the supply of group I commodities), which can be indexed either monotonically by the severity of supply rationing, or by the ratio of group II prices (say, their sum) to group I prices.

Borrowing that result, one could state the following about the model studied above. Call "group I" the set of markets on which prices are set by firms or unions entertaining kinky demand perceptions.

Conjecture 1 With almost every Keynes-Negishi equilibrium, one can associate a continuum of other Keynes-Negishi equilibria with the same group I prices but different group I quantities. ${ }^{29}$

This conjecture, if correct, would come a long way towards delivering the very application pursued in Negishi (1979), if one thinks about selection of an element from the continuum as triggered by an aggregate demand shock. The same conjecture would also come a long way toward establishing a dual proposition: exogenous shocks affecting the prices of group II commodities (an oil shock?) could result in quantity adjustments at unchanged prices for group I commodities.

This dual proposition assumes that the location of the kinks does not move with group II prices; as remarked in Subsubsection 7.2.2, there are alternative possibilities.

Although aggregate demand shocks provide a privileged application of GET with demand kinks, some others come to mind. Again, the analogy with fixed prices is instructive, as models with price rigidities have proved instructive to study such issues as public sector pricing (Drèze 1984) or the stability of tatonnement (Drèze 1999), as well as non-tâtonnement (Drèze 1991) processes. "To tell the story of Keynes-Negishi equilibrium, therefore, one must talk of its applications as well as of its pure theory.” (Negishi, 1972, p. vii)

\section{Appendix}

Proof Of Lemma 2: Let $s^{-}$denote the sign vector with all components -1 , and $s^{+}$the sign vector with all components +1 . Consider an arbitrary sign vector $s \in \mathcal{S}$. When $y^{j}$ belongs to

$$
\mathbb{R}^{L}\left(\bar{y}^{j}, s\right) \cap \mathbb{R}^{L}\left(\bar{y}^{j}, s^{-}\right)=\left\{y \in \mathbb{R}^{L}\left(\bar{y}^{j}, s^{-}\right) \mid y_{l}=\bar{y}_{l}^{j} \quad \text { when } \quad s_{l}=+1\right\},
$$

then $H^{j s}(\bar{a}, \bar{p})\left(y^{j}-\bar{y}^{j}\right)$ and $H^{j-}(\bar{a}, \bar{p})\left(y^{j}-\bar{y}^{j}\right)$ should coincide. It then follows that $H_{\cdot l}^{j s}(\bar{a}, \bar{p})=H_{\cdot l}^{j-}(\bar{a}, \bar{p})$ if $s_{l}=-1$.

\footnotetext{
${ }^{29}$ Almost every: more precisely, all equilibria with group I prices in the relative interior of the relevant pricing rules.
} 
From the fact that $H^{j s}(\bar{a}, \bar{p})\left(y^{j}-\bar{y}^{j}\right)$ and $H^{j+}(\bar{a}, \bar{p})\left(y^{j}-\bar{y}^{j}\right)$ should coincide for $y^{j} \in \mathbb{R}^{L}\left(\bar{y}^{j}, s\right) \cap$ $\mathbb{R}^{L}\left(\bar{y}^{j}, s^{+}\right)$, we derive that $H_{\cdot l}^{j s}(\bar{a}, \bar{p})=H_{\cdot l}^{j+}(\bar{a}, \bar{p})$ if $s_{l}=+1$.

Proof of Lemma 3: The production plan $\bar{y}^{j}$ maximizes profits given market data $(\bar{a}, \bar{p})$ if and only if for every $s \in \mathcal{S}, \bar{y}^{j}$ maximizes profits on $\bar{Y}^{j s}:=Y^{j} \cap \mathbb{R}^{L}\left(\bar{y}^{j}, s\right)$. Similar to (2), FOC for maximal profits at $\bar{y}^{j}$ on $\bar{Y}^{j s}$ require the existence of a vector $\bar{q}^{j s}$ in $N_{\bar{Y}^{j s}}^{j}\left(\bar{y}^{j}\right)$, the normal cone to $\bar{Y}^{j s}$ at $\bar{y}^{j}$, such that

$$
\bar{p}+H^{j s}(\bar{a}, \bar{p})^{\top} \bar{y}^{j}=\bar{q}^{j s}
$$

Because $N_{\bar{Y}^{j s}}^{j}\left(\bar{y}^{j}\right)=N^{j}\left(\bar{y}^{j}\right)-\mathbb{R}^{L}(0, s)$, the result follows.

Proof of Lemma 4: To show that (7) and (8) are sufficient, define, for $s \in \mathcal{S}, q^{j s}=q^{j}$ and $\mu^{j s}$ by

$$
\mu_{l}^{j s}= \begin{cases}q_{l}^{j}-H_{\cdot l}^{j-}(\bar{a}, \bar{p})^{\top} \bar{y}^{j}-p_{l}^{j} \leq 0, & \text { if } s_{l}=-1 \\ q_{l}^{j}-H_{\cdot l}^{j+}(\bar{a}, \bar{p})^{\top} \bar{y}^{j}-p_{l}^{j} \geq 0, & \text { if } s_{l}=+1\end{cases}
$$

Then $q^{j s}$, now independent of $s$, and $\mu^{j s}$ fulfill the necessary and sufficient conditions of Lemma 2.

Proof of Lemma 5: We start with a proof of P.2. Consider first the case where

$$
1+\iota^{\top} \bar{H}^{j}\left(y^{j} ; \bar{a}, \bar{p}\right)^{\top} y^{j} \geq 0
$$

Then, $\bar{q}^{j} \max \left(0,1+\iota^{\top} \bar{H}^{j}\left(y^{j} ; \bar{a}, \bar{p}\right)^{\top} y^{j}\right)$ equals a non-negative multiple of $\bar{q}^{j}$, so belongs to $N^{j}\left(y^{j}\right)$, and $p^{j}$ satisfies (7) and (8) with $\bar{y}^{j}$ replaced by $y^{j}$, so $y^{j}$ maximizes profits for an inverse demand function given by

$$
H^{j s}\left(y^{j} ; \bar{a}, \bar{p}\right)\left(\tilde{y}^{j}-y^{j}\right)+p^{j}, \quad s \in \mathcal{S}, \tilde{y}^{j} \in \mathbb{R}^{L}\left(y^{j}, s\right) .
$$

In particular, $p^{j^{\top}} y^{j} \geq 0$, because the firm can achieve zero profits by remaining inactive.

The second case is where

$$
1+\iota^{\top} \bar{H}^{j}\left(y^{j} ; \bar{a}, \bar{p}\right)^{\top} y^{j}<0
$$

Then $\left(-\iota^{\top} \bar{H}^{j}\left(y^{j} ; \bar{a}, \bar{p}\right)^{\top} y^{j}\right) p^{j}$ satisfies (7) and (8), with $\bar{y}^{j}$ replaced by $y^{j}$ and $q^{j}$ equal to the zero-vector, an element of $N^{j}\left(y^{j}\right)$, so by the same reasoning as before,

$$
\left(-\iota^{\top} \bar{H}^{j}\left(y^{j} ; \bar{a}, \bar{p}\right)^{\top} y^{j}\right) p^{j^{\top}} y^{j} \geq 0
$$

Because $-\iota^{\top} \bar{H}^{j}\left(y^{j} ; \bar{a}, \bar{p}\right)^{\top} y^{j}>1$, it follows that $p^{j^{\top}} y^{j} \geq 0$, as desired.

Now we turn to the proof of P.1. Where unnecessary, we omit the superscript $j$ and the reference to $(\bar{a}, \bar{p})$.

The proof is a tedious sequence of elementary steps, mostly relying on properties of upper hemi-continuous correspondences as collected in Hildenbrand (1974). To structure the proof, we introduce the following definitions 
and notation:

$$
\begin{aligned}
& \bar{N}^{j}: \partial Y^{j} \rightarrow \Delta, \bar{N}^{j}\left(y^{j}\right):=N^{j}\left(y^{j}\right) \cap \Delta ; \\
& \omega^{j-}: \partial Y^{j} \times A \times \Delta \times \Delta \rightarrow S, \\
& \omega^{j-}\left(y^{j}, \bar{a}, \bar{p}, \bar{q}^{j}\right):=\left\{p^{j} \in S \mid\right. \\
& \left.\bar{q}^{j} \max \left(0,1+\iota^{\top} \bar{H}^{j}\left(y^{j} ; \bar{a}, \bar{p}\right)^{\top} y^{j}\right)-\frac{H^{j-}\left(y^{j} ; \bar{a}, \bar{p}\right)^{\top} y^{j}}{\max \left(1,-\iota^{\top} \bar{H}^{j}\left(y^{j} ; \bar{a}, \bar{p}\right)^{\top} y^{j}\right)} \leq p^{j}\right\} ; \\
& \omega^{j+}: \partial Y^{j} \times A \times \Delta \times \Delta \rightarrow S, \\
& \omega^{j+}\left(y^{j}, \bar{a}, \bar{p}, \bar{q}^{j}\right):=\left\{p^{j} \in S \mid\right. \\
& \left.p^{j} \leq \bar{q}^{j} \max \left(0,1+\iota^{\top} \bar{H}^{j}\left(y^{j} ; \bar{a}, \bar{p}\right)^{\top} y^{j}\right)-\frac{H^{j+}\left(y^{j} ; \bar{a}, \bar{p}\right)^{\top} y^{j}}{\max \left(1,-\iota^{\top} \bar{H}^{j}\left(y^{j} ; \bar{a}, \bar{p}\right)^{\top} y^{j}\right)}\right\} ; \\
& \omega^{j}: \partial Y^{j} \times A \times \Delta \times \Delta \rightarrow S, \\
& \omega^{j}\left(y^{j}, \bar{a}, \bar{p}, \bar{q}^{j}\right)=\omega^{j-}\left(y^{j}, \bar{a}, \bar{p}, \bar{q}^{j}\right) \cap \omega^{j+}\left(y^{j}, \bar{a}, \bar{p}, \bar{q}^{j}\right) ; \\
& \varphi^{j}: \partial Y^{j} \times A \times \Delta \rightarrow S \text {, } \\
& \varphi^{j}=\omega^{j} \circ\left(I \times \bar{N}^{j}\right), \\
& \varphi^{j}\left(y^{j} ; \bar{a}, \bar{p}\right)=\left\{p^{j} \in \mathbb{R}^{L} \mid \exists \bar{q}^{j} \in \bar{N}^{j}\left(y^{j}\right): p^{j} \in \omega^{j}\left(y^{j}, \bar{a}, \bar{p}, \bar{q}^{j}\right)\right\},
\end{aligned}
$$

where the symbol $\circ$ stands for composition of correspondences and $I: \partial Y^{j} \times A \times \Delta \rightarrow \partial Y^{j} \times \mathrm{A} \times \Delta$ denotes the identity.

We must show that $\varphi^{j}$ is non-empty, convex, and compact valued and upper hemi-continuous with respect to $\left(y^{j}, \bar{a}, \bar{p}\right)$ over compact sets in $\partial Y^{j} \times A \times \Delta$; namely, that $\varphi^{j}$ satisfies P.1.

The proof is constructed "bottom-up", starting with properties of $\bar{N}(y)$, then turning to $\omega^{-}$and $\omega^{+}$, next to $\omega$; the final step, concerning $\varphi$, is then in sight.

Step 1 The set $N(y)$ is a closed convex cone of $\mathbb{R}_{+}^{L}$ with vertex 0 . Indeed, under free disposal $\left(Y^{j}+\mathbb{R}_{-}^{L} \subset Y^{j}\right)$ and $0 \in Y^{j}, q \in N(y)$ implies $q \in \mathbb{R}_{+}^{L}$. Also, $N$ is closed. So, $\bar{N}$ is non-empty, convex and compact valued, and closed.

Step 2 Regarding $\omega^{-}(y, \bar{a}, \bar{p}, \bar{q})$, three cases must be distinguished, according as $1+\iota^{\top} \bar{H}(y ; \bar{a}, \bar{p})^{\top} y$ is positive (Step 2.1), negative (Step 2.2) or zero (Step 2.3). In all cases, for $y$ and $\bar{a}$ in a closed cube of $\mathbb{R}^{L}$ with finite length and for $\bar{p} \in \Delta$, Assumption A.3' implies that $H^{-}(y ; \bar{a}, \bar{p})$ and $H^{+}(y ; \bar{a}, \bar{p})$ are continuous in $(y, \bar{a}, \bar{p})$ and bounded.

Step 2.1 Let $\quad \omega^{-}(y, \bar{a}, \bar{p}, \bar{q})=\left\{p \in \mathbb{R}^{L} \mid \bar{q} \max \left(0,1+\iota^{\top} \bar{H}(y ; \bar{a}, \bar{p}) y\right)^{\top}-H^{-}(y ; \bar{a}, \bar{p})^{\top} y \leq p\right\}$, with $1+\iota^{\top} \bar{H}(\cdot)^{\top} y>0$ and with $\bar{q}=\bar{q}(y) \in \bar{N}(y)$, a convex, compact set; then $\omega^{-}(\cdot)$ is non-empty, convex, and closed valued. For $y$ and $\bar{a}$ in a closed cube of $\mathbb{R}^{L}$ with finite length, $\omega^{-}$is bounded from below.

Step 2.2 Let instead $\omega^{-}(y, \bar{a}, \bar{p}, \bar{q})=\left\{p \in \mathbb{R}^{L} \mid \frac{-H^{-}(y ; \bar{a}, \bar{p})^{\top} y}{-\iota^{\top} \bar{H}(y ; \bar{a}, \bar{p})^{\top} y} \leq p\right\}$; then $\omega^{-}$is non-empty, convex, and closed valued; for $y$ and $\bar{a}$ in a closed cube of $\mathbb{R}^{L}$ with finite length, $\omega^{-}$is bounded from below.

Step 2.3 At $1+\iota^{\top} \bar{H}(y ; \bar{a}, \bar{p})^{\top} y=0$, the sets of admissible values of $p$ obtained in Step 2.1 and Step 2.2 are identical, and $\omega^{-}$is closed.

Step 3 The reasoning in Step 2 applies unchanged to $\omega^{+}(y, \bar{a}, \bar{p}, \bar{q})$, except that this time $\omega^{+}$is bounded from above.

Using coordinate-wise the lower of the two bounds in Step 2.1 and Step 2.2, we may write $p \geq \underline{p}^{-}$, whenever $p$ belongs to $\omega^{-}(y, \bar{a}, \bar{p}, \bar{q})$ for $(y, \bar{a})$ in a closed cube of $\mathbb{R}^{L}$ with finite length; similarly, $p \leq \bar{p}^{+}$, whenever $p$ belongs to $\omega^{+}(y, \bar{a}, \bar{p}, \bar{q})$ for $(y, \bar{a})$ in a closed cube of $\mathbb{R}^{L}$ with finite length. We have noted in Section 3.1 that the intersection of $\omega^{-}(\cdot)$ and $\omega^{+}(\cdot)$ (i.e. $\left.\omega(\cdot)\right)$ is non-empty. So, we 
may define:

$$
\begin{aligned}
& \bar{\omega}^{-}(y, \bar{a}, \bar{p}, \bar{q}):=\left\{p \in \mathbb{R}^{L} \mid p \in \omega^{-}(y, \bar{a}, \bar{p}, \bar{q}), p \leq \bar{p}^{+}\right\}, \\
& \bar{\omega}^{+}(y, \bar{a}, \bar{p}, \bar{q}):=\left\{p \in \mathbb{R}^{L} \mid p \in \omega^{+}(y, \bar{a}, \bar{p}, \bar{q}), p \geq \underline{p}^{-}\right\} .
\end{aligned}
$$

These correspondences are now compact valued. It follows that $\bar{\omega}^{-}$and $\bar{\omega}^{+}$are upper hemi-continuous, and so is their intersection $\omega(y, \bar{a}, \bar{p}, \bar{q})$ : see proposition 2 of Hildenbrand (1974). Furthermore, it is immediate that $\omega$ is non-empty, convex, and compact valued.

Step 4 We turn now to $\varphi$. As a composition of two upper hemi-continuous correspondences, $\varphi$ is itself upper hemi-continuous (Hildenbrand (1974) corollary on p. 22). Also, it is non-empty and compact valued, because $\omega$ is non-empty and compact valued for all $\bar{q} \in \bar{N}(y)$ and $\bar{N}(y)$ is itself non-empty and compact valued. It is convex valued, because $\bar{N}(y)$ is convex valued and $\bar{q}$ enters linearly in $\bar{\omega}^{-}$and $\bar{\omega}^{+}$, hence in $\omega$. So $\varphi$ is non-empty, convex and compact valued, and upper hemi-continuous. It satisfies P.1 and P.2, for $(\bar{y}, \bar{a}, \bar{p})$ in compact sets.

Proof of Theorem 3: Suppose that there exist $\tilde{y}^{k}$ and associated $\tilde{p}=\pi^{k}\left(\tilde{y}^{k} ; \bar{a}, \bar{p}\right)$ and $\left(\tilde{x}^{h}\right)_{h \in \mathcal{H}^{k}}$ satisfying (17)(19). We define $d y^{k}=\tilde{y}^{k}-\bar{y}^{k}, d p=\tilde{p}-\bar{p}$, and $d x^{h}=\tilde{x}^{h}-\bar{x}^{h}, h \in \mathcal{H}^{k}$. Then (19) implies $\bar{\lambda}^{h^{\top}} d x^{h}>0$ for all $h \in \mathcal{H}^{k}$, with

$$
d x^{h k}=\frac{d \sigma^{h k}}{d y^{k k}} d y^{k k}
$$

as per (18); thus:

$$
k_{\bar{p}}^{\top} d^{k} x^{h}+\bar{\lambda}^{h k^{\top}} \frac{d \sigma^{h k}}{d y^{k k}} d y^{k k}>0, \forall h \in \mathcal{H}^{k}
$$

so

$$
\sum_{h \in \mathcal{H}^{k}} k_{\bar{p}}^{\top} d^{k} x^{h}+\bar{\lambda}^{h k^{\top}} \frac{d \sigma^{h k}}{d y^{k k}} d y^{k k}>0 .
$$

It holds that

$$
\begin{aligned}
\sum_{h \in \mathcal{H}^{k}} k_{\bar{p}}{ }^{\top} d^{k} x^{h} & \leq \sum_{h \in \mathcal{H}^{k}}\left({ }^{k} \bar{p}^{\top k} \tilde{x}^{h}-{ }^{k} \tilde{p}^{\top k} \tilde{x}^{h}+\bar{p}^{k^{\top}} \bar{x}^{h k}-\tilde{p}^{k^{\top}} \tilde{x}^{h k}\right) \\
& =\sum_{h \in \mathcal{H}^{k}}\left(-\bar{p}^{k^{\top}} d x^{h k}-d p^{k^{\top}} \tilde{x}^{h k}\right) \\
& =\bar{p}^{k^{\top}} d y^{k k}+\tilde{y}^{k k^{\top}} d p^{k} \\
& =\bar{p}^{k^{\top}} d y^{k k}+\bar{y}^{k k^{\top}} \frac{d \pi^{k k}}{d y^{k k}} d y^{k k}+d y^{k k} \frac{d \pi^{k k}}{d y^{k k}} d y^{k k} \\
& \leq\left[\bar{p}^{k^{\top}}+\bar{y}^{k k^{\top}} \frac{d \pi^{k k}}{d y^{k k}}\right] d y^{k k}
\end{aligned}
$$

where the first inequality uses (17) and the last inequality the assumption that $H^{k s}, s \in \mathcal{S}$, is NSD. Combining (33) and (34) results in

$$
\left[\bar{p}^{k^{\top}}+\bar{y}^{k k^{\top}} \frac{d \pi^{k k}}{d y^{k k}}+\sum_{h \in \mathcal{H}^{k}} \bar{\lambda}^{h k^{\top}} \frac{d \sigma^{h k}}{d y^{k k}}\right] d y^{k k}>0 .
$$

There is $l \in \mathcal{L}^{k}$ such that $\left(\bar{p}_{l}^{k}+\bar{y}^{k k^{\top}} \frac{d \pi_{l}^{k}}{d y^{k k}}+\sum_{h \in \mathcal{H}^{k}} \bar{\lambda}^{h k^{\top}} \frac{d \sigma_{l}^{h}}{d y_{l}^{k}}\right) d y_{l}^{k}>0$. For $d y_{l}^{k}>0$, implying $\bar{y}_{l}^{k}<\hat{y}_{l}^{k}$, implies 


$$
\bar{p}_{l}^{k}>\sum_{h}-\bar{\lambda}^{h k^{\top}} \frac{d \sigma_{l}^{h}}{d y_{l}^{k}}-H_{\cdot l}^{k k+}(\bar{a}, \bar{p})^{\top} \bar{y}^{k k},
$$

contradicting the right-hand side inequality in (20). For $d y_{l}^{k}<0$, implying $\bar{y}_{l}^{k}>0$, (35) implies

$$
\bar{p}_{l}^{k}<\sum_{h}-\bar{\lambda}^{h k^{\top}} \frac{d \sigma_{l}^{h}}{d y_{l}^{k}}-H_{\cdot l}^{k k-}(\bar{a}, \bar{p})^{\top} \bar{y}^{k k},
$$

contradicting the left-hand side inequality in (20).

Proof of Lemma 6: The first statement of the lemma follows in the same way as in Section 3.

We show next that every $p^{j}$ satisfying (26) yields $p^{j^{\top}} R^{+}\left(y^{j}\right) y^{j} \geq 0$. Consider first the case where

$$
\mu^{j}\left(y^{j} ; \bar{a}, \bar{p}\right) \leq 1
$$

Then, $\max \left(0,1-\mu^{j}\left(y^{j} ; \bar{a}, \bar{p}\right)\right) \bar{q}^{j}$ equals a non-negative multiple of $\bar{q}^{j}$, so belongs to $N^{j}\left(y^{j}\right)$, and $p^{j}$ satisfies the sufficient conditions (24) and (25), with $\bar{y}^{j}$ replaced by $y^{j}$, so $y^{j}$ maximizes profits for an inverse demand function given by

$$
H^{j s}\left(y^{j} ; \bar{a}, \bar{p}\right)\left(\tilde{y}^{j}-y^{j}\right)+p^{j}, \quad s \in \mathcal{S}, \tilde{y}^{j} \in \mathbb{R}^{L N}\left(y^{j}, s\right)
$$

In particular, $p^{j^{\top}} R^{+}\left(y^{j}\right) y^{j} \geq 0$, because the firm can achieve zero profits by remaining inactive.

The second case is where

$$
\mu^{j}\left(y^{j} ; \bar{a}, \bar{p}\right)>1
$$

Then $\mu^{j}\left(y^{j} ; \bar{a}, \bar{p}\right) p^{j}$ satisfies the adjusted sufficient conditions (24) and (25) with $\bar{y}^{j}$ replaced by $y^{j}$ and $q^{j}$ equal to the zero-vector, an element of $N^{j}\left(y^{j}\right)$, so by the same reasoning as before,

$$
\mu^{j}\left(y^{j} ; \bar{a}, \bar{p}\right) p^{j^{\top}} R^{+}\left(y^{j}\right) y^{j} \geq 0
$$

Because $\mu^{j}\left(y^{j} ; \bar{a}, \bar{p}\right)>1$, it follows that $p^{j^{\top}} R^{+}\left(y^{j}\right) y^{j} \geq 0$, as desired.

Proof of Theorem 5: ${ }^{30}$ Given some $r \in \mathbb{R}_{+}^{N}$, we want to show existence of a tuple $\left(p, r,\left(x^{h}\right)_{h \in \mathcal{H}},\left(y^{j}\right)_{j \in \mathcal{J}},\left(y^{k}\right)_{k \in \mathcal{K}}\right)$ satisfying the properties stated in Definition 5.

The boundedness of the set $A$ allows us to replace consumption sets $X^{h}$ by $\bar{X}^{h}=X^{h} \cap C$, where $C$ is a closed cube in $\mathbb{R}^{L N}$ with length $c$ centered at the origin. The cube $C$ is such that $a \in A$ implies, for every $h \in \mathcal{H}, x^{h}$ in the interior of $C$, and for every $j \in \mathcal{J}, y^{j}$ in the interior of $C$. We define the relevant part of the production set by $\bar{Y}^{j}=\left\{y^{j} \in \partial Y^{j} \mid y^{j}+c \iota \in \mathbb{R}_{+}^{L N}\right\}$. For $j \in \mathcal{J}$, we define the homeomorphism $g^{j}$ between $\Delta^{L N}$ and $\bar{Y}^{j}$ as follows. For $y^{j} \in \bar{Y}^{j}$,

$$
g^{j^{-1}} \cdot\left(y^{j}\right)=\frac{y^{j}+c \iota}{\sum_{l_{s_{t} \in \mathcal{L} \times \mathcal{N}}}\left(y_{l_{s_{t}}}^{j}+c\right)}:=\alpha^{j} .
$$

Then $g^{j^{-1}}\left(y^{j}\right) \gg 0$ if and only if $y^{j}+c \iota \gg 0$.

For $j \in \mathcal{J}$, the set

$$
\left\{p^{j} \in S^{L N} \mid \exists y^{j} \in \bar{Y}^{j}, \exists a \in A, \exists p \in \Delta^{L N} \text { such that } p^{j} \in \varphi^{j}\left(y^{j} ; a, p\right)\right\}
$$

is bounded, so it is contained in a compact, convex set $S^{j}$.

\footnotetext{
30 The logical structure of this step is parallel to that of the proof of theorem 2 in Dehez and Drèze (1988).
} 
For $k \in \mathcal{K}$, let $\tilde{\varphi}^{k}\left(y^{k} ; a, p\right)$ be defined as the subset of $\varphi^{k}\left(y^{k} ; a, p\right)$ as defined in (32) that satisfies the lower bound on $p_{l_{s_{t}}}^{k}$ for $l s_{t} \in \mathcal{L}^{k} \times \mathcal{N}$ such that $y_{l_{s_{t}}}^{k}=0$, and the upper bound on $p_{l_{s_{t}}}^{k}$ for $l s_{t} \in \mathcal{L}^{k} \times \mathcal{N}$ such that $y_{l_{s_{t}}}^{k}=\hat{y}_{l_{s_{t}}}^{k}$. The set

$$
\left\{p^{k} \in \mathbb{R}^{L N} \mid \exists y^{k} \in \bar{Y}^{k}, \exists a \in A, \exists p \in \Delta^{L N} \text { such that } p^{k} \in \tilde{\varphi}^{k}\left(y^{k} ; a, p\right)\right\}
$$

is bounded, so it is contained in a compact, convex set $S^{k}$.

We define the correspondence, whose fixed points are equilibria, $\Phi$ from $\Delta^{L N} \times \prod_{j \in \mathcal{J}} S^{j} \times \prod_{k \in \mathcal{K}} S^{k} \times$ $\Delta^{J L N} \times \prod_{k} Y^{k} \times \prod_{h} \bar{X}^{h}$ into itself by:

$$
\begin{aligned}
\Phi\left(p, p^{\mathcal{J}}, p^{\mathcal{K}}, \alpha, y^{\mathcal{K}}, x\right)= & \mu(x, \alpha) \times \prod_{j} \bar{\varphi}^{j}(p, \alpha, x) \times \prod_{k} \bar{\varphi}^{k}\left(p, \alpha, y^{k}, x\right) \\
& \times \prod_{j} \beta^{j}\left(p, p^{j}, \alpha^{j}\right) \times \prod_{k} \beta^{k}\left(p, p^{k}, y^{k}\right) \times \prod_{h} \xi^{h}\left(p, \alpha, y^{\mathcal{K}}, x\right) .
\end{aligned}
$$

Here, market prices are determined through the market price correspondence $\mu: \prod_{h} \bar{X}^{h} \times \Delta^{J L N} \rightarrow \Delta^{L N}$ defined by

$$
\mu(x, \alpha)=\arg \max _{p \in \Delta^{L N}} p^{\top}\left(\sum_{h \in \mathcal{H}} x^{h}-\sum_{h \in \mathcal{H}} e^{h}-\sum_{j \in \mathcal{J}} g^{j}\left(\alpha^{j}\right)\right)
$$

The prices of firm $j$ are determined through the correspondence $\bar{\varphi}^{j}: \Delta^{L N} \times \Delta^{J L N} \times \prod_{h} \bar{X}^{h} \rightarrow S^{j}$ defined by

$$
\bar{\varphi}^{j}(p, \alpha, x)=\varphi^{j}\left(g^{j}\left(\alpha^{j}\right) ; \rho\left(x,\left(g^{j}\left(\alpha^{j}\right)\right) j \in \mathcal{J}, p\right),\right.
$$

where $\varphi^{j}$ is defined in (26) and $\rho$ is the projection on $A$.

The prices of union $k$ are determined through the correspondence $\bar{\varphi}^{k}: \Delta^{L N} \times \Delta^{J L N} \times Y^{k} \times \prod_{h} \bar{X}^{h} \rightarrow S^{k}$ defined by

$$
\bar{\varphi}^{k}\left(p, \alpha, y^{k}, x\right)=\tilde{\varphi}^{k}\left(y^{k} ; \rho\left(x,\left(g^{j}\left(\alpha^{j}\right)\right)_{j \in \mathcal{J}, p)}\right.\right.
$$

The quantity adjustment correspondence of firm $j$ is a function $\beta^{j}: \Delta^{L N} \times S^{j} \times \Delta^{L N} \rightarrow \Delta^{\mathrm{LN}}$ defined by

$$
\beta_{l^{\prime} s_{t^{\prime}}^{\prime}}^{j}\left(p, p^{j}, \alpha^{j}\right)=\max \left\{0, \alpha_{l^{\prime} s_{t^{\prime}}^{\prime}}^{j}+p_{l^{\prime} s_{t^{\prime}}^{\prime}}-p_{l^{\prime} s_{t^{\prime}}^{\prime}}^{j}\right\} / \sum_{l s_{t}} \max \left\{0, \alpha_{l_{s}}^{j}+p_{l s_{t}}-p_{l_{s_{t}}}^{j}\right\}, \quad l^{\prime} s_{t^{\prime}}^{\prime} \in \mathcal{L} \times \mathcal{N}
$$

The quantity adjustment correspondence of union $k$ is a function $\beta^{k}: \Delta^{L N} \times S^{k} \times Y^{k} \rightarrow \mathrm{Y}^{\mathrm{k}}$ defined by

$$
\begin{aligned}
& \beta_{l s_{t}}^{k}\left(p, p^{k}, y^{k}\right)=\min \left\{\max \left\{0, y_{l s_{t}}^{k}+p_{l s_{t}}-p_{l s_{t}}^{k}\right\}, \hat{y}_{l s_{t}}^{k}\right\}, \quad l s_{t} \in \mathcal{L}^{k} \times \mathcal{N}, \\
& \beta_{l s_{t}}^{k}\left(p, p^{k}, y^{k}\right)=0, \quad l s_{t} \in(\mathcal{L} \times \mathcal{N}) \backslash\left(\mathcal{L}^{k} \times \mathcal{N}\right)
\end{aligned}
$$

The demand correspondence of household $h, \xi^{h}: \Delta^{L N} \times \Delta^{L N} \times \prod_{k} Y^{k} \times \prod_{h} \bar{X}^{h} \rightarrow \bar{X}^{h}$ is defined as follows using a technique introduced in Greenberg (1977). Given $\left(p, \alpha, y^{\mathcal{K}}, x\right)$, define the modified budget set $\gamma^{h}\left(p, \alpha, y^{\mathcal{K}}, x\right)$ by

$$
\begin{aligned}
\gamma^{h}\left(p, \alpha, y^{\mathcal{K}}, x\right) & =\left\{\bar{x}^{h} \in \bar{X}^{h} \mid p^{\top} R^{\circ}\left(\bar{z}^{h}\right) \bar{z}^{h} \leq \max \left\{0, \sum_{j \in \mathcal{J}} \theta^{h j} \hat{v}^{j}+\theta^{h b} \hat{v}^{b}\right\},\right. \\
\bar{x}^{h k} & \left.=\sigma^{h k}\left(y^{k}\right) \quad \text { if } h \in \mathcal{H}^{k}\right\},
\end{aligned}
$$

where $\quad \hat{v}^{j}=p^{\top} R^{+}\left(g^{j}\left(\alpha^{j}\right)\right) g^{j}\left(\alpha^{j}\right), \quad j \in \mathcal{J} \quad$ and $\quad \hat{v}^{b}=\sum_{h \in \mathcal{H}} p^{\top}\left(I-R^{\circ}\left(z^{h}\right)\right) z^{h}+\sum_{j \in \mathcal{J}} p^{\top}(I-$ $\left.R^{+}\left(g^{j}\left(\alpha^{j}\right)\right)\right) g^{j}\left(\alpha^{j}\right)$. Because out of equilibrium the sum of the dividends can be negative, we use the modified budget set, and replace a negative sum of dividends by zero. If $\left(p, \alpha, y^{\mathcal{K}}, x\right)$ is such that there is $\bar{x}^{h} \in \gamma^{h}\left(p, \alpha, y^{\mathcal{K}}, x\right)$ with $p^{\top} R^{\circ}\left(\bar{z}^{h}\right) \bar{z}^{h}<\max \left\{0, \sum_{j \in \mathcal{J}} \theta^{h j} \hat{v}^{j}+\theta^{h b} \hat{v}^{b}\right\}$, then $\xi^{h}\left(p, \alpha, y^{\mathcal{K}}, x\right)$ is defined as 
the set of $\succeq_{h}$-maximizers on $\gamma^{h}\left(p, \alpha, y^{\mathcal{K}}, x\right)$. Otherwise, we define $\xi^{h}\left(p, \alpha, y^{\mathcal{K}}, x\right)=\gamma^{h}\left(p, \alpha, y^{\mathcal{K}}, x\right)$. These definitions lead to upper hemi-continuous correspondences $\xi^{h}$.

The correspondence $\Phi$ satisfies the assumptions of Kakutani's fixed point theorem, so has a fixed point $\left(\bar{p}, \bar{p}^{\mathcal{J}}, \bar{p}^{\mathcal{K}}, \bar{\alpha}, \bar{y}^{\mathcal{K}}, \bar{x}\right)$ inducing production bundles $g^{j}\left(\bar{\alpha}^{j}\right)$ for a firm $j \in \mathcal{J}$ and total excess demand

$$
\bar{\zeta}=\sum_{h \in \mathcal{H}} \bar{x}^{h}-\sum_{h \in \mathcal{H}} e^{h}-\sum_{j \in \mathcal{J}} g^{j}\left(\bar{\alpha}^{j}\right) .
$$

Clearly, $g^{j}\left(\bar{\alpha}^{j}\right) \in \partial Y^{j}, \quad j \in \mathcal{J}$, and the following conditions are satisfied:

$$
\begin{gathered}
p^{\top} \bar{\zeta} \leq \bar{p}^{\top} \bar{\zeta}, \quad p \in \Delta^{L N}, \\
\bar{p}^{j} \in \bar{\varphi}^{j}(\bar{p}, \bar{\alpha}, \bar{x}), \quad j \in \mathcal{J}, \\
\bar{p}^{k} \in \bar{\varphi}^{k}\left(\bar{p}, \bar{\alpha}, \bar{y}^{k}, \bar{x}\right), \quad k \in \mathcal{K}, \\
\bar{\alpha}^{j}=\beta^{j}\left(\bar{p}, \bar{p}^{j}, \bar{\alpha}^{j}\right), \quad j \in \mathcal{J}, \\
\bar{y}^{k}=\beta^{k}\left(\bar{p}, \bar{p}^{k}, \bar{y}^{k}\right), \quad k \in \mathcal{K}, \\
\bar{x}^{h} \in \xi^{h}\left(\bar{p}, \bar{\alpha}, \bar{y}^{\mathcal{K}}, \bar{x}\right), \quad h \in \mathcal{H} .
\end{gathered}
$$

For $j \in \mathcal{J}$, we define $\bar{\beta}^{j}=\sum_{l_{s_{t}} \in \mathcal{L} \times \mathcal{N}} \max \left\{0, \bar{\alpha}_{l_{s_{t}}}^{j}+\bar{p}_{l_{s_{t}}}-\bar{p}_{l_{s_{t}}}^{j}\right\}$. Then, (39) implies

$$
\bar{\beta}^{j} \bar{\alpha}_{l_{s_{t}}}^{j} \geq \bar{\alpha}_{l_{s_{t}}}^{j}+\bar{p}_{l_{s_{t}}}-\bar{p}_{l_{s_{t}}}^{j}, \quad j \in \mathcal{J},
$$

with equality whenever $\bar{\alpha}_{l s_{t}}^{j}>0$. Multiplying both sides of $(41)$ by $R_{l_{t}, l s_{t}}^{+}\left(g^{j}\left(\bar{\alpha}^{j}\right)\right) \bar{\alpha}_{l s_{t}}^{j}$ and summing over all $l_{s_{t}}$, we get

$$
\left(\bar{\beta}^{j}-1\right) \bar{\alpha}^{j^{\top}} R^{+}\left(g^{j}\left(\bar{\alpha}^{j}\right)\right) \bar{\alpha}^{j}=\left(\bar{p}-\bar{p}^{j}\right)^{\top} R^{+}\left(g^{j}\left(\bar{\alpha}^{j}\right)\right) \bar{\alpha}^{j},
$$

where $\bar{\beta}^{j} \geq 1$ and $\bar{\alpha}^{j^{\top}} R^{+}\left(g^{j}\left(\bar{\alpha}^{j}\right)\right) \bar{\alpha}^{j} \geq 0$. Therefore, we obtain that

$$
\left(\bar{p}-\bar{p}^{j}\right)^{\top} R^{+}\left(g^{j}\left(\bar{\alpha}^{j}\right)\right) \bar{\alpha}^{j} \geq 0 \quad \text { for all } j \in \mathcal{J} .
$$

By definition of $g^{j}$, there exists $\mu>0$ and $v>0$ such that

$$
g^{j}\left(\bar{\alpha}^{j}\right)=\mu \bar{\alpha}^{j}-v \iota
$$

We then have

$$
\left(\bar{p}-\bar{p}^{j}\right)^{\top} R^{+}\left(g^{j}\left(\bar{\alpha}^{j}\right)\right) g^{j}\left(\bar{\alpha}^{j}\right)=\left(\bar{p}-\bar{p}^{j}\right)^{\top} R^{+}\left(g^{j}\left(\bar{\alpha}^{j}\right)\right) \mu \bar{\alpha}^{j}-\left(\bar{p}-\bar{p}^{j}\right)^{\top} R^{+}\left(g^{j}\left(\bar{\alpha}^{j}\right)\right) \nu \iota .
$$


This expression is non-negative, since the first term is non-negative by (42) and the term subtracted is non-positive, because $g_{l_{s_{t}}}^{j}\left(\bar{\alpha}^{j}\right)>0$ implies $\bar{\alpha}_{l_{s_{t}}}^{j}>0$, so by (39), $0 \leq\left(\bar{\beta}^{j}-1\right) \bar{\alpha}_{l_{s_{t}}}^{j}=\bar{p}_{l_{s_{t}}}-\bar{p}_{l_{s_{t}}}^{j}$ and

$$
\begin{aligned}
& \left(\bar{p}-\bar{p}^{j}\right)^{\top} R^{+}\left(g^{j}\left(\bar{\alpha}^{j}\right)\right) \iota=\sum_{l s_{t} \mid g_{l_{s}}^{j}\left(\bar{\alpha}^{j}\right)>0} R_{l_{t}, l s_{t}}^{+}\left(g^{j}\left(\bar{\alpha}^{j}\right)\right)\left(\bar{p}-\bar{p}_{l_{s_{t}}}^{j}\right) \\
& +\sum_{l_{s_{t} \mid g_{l_{t}}^{j}\left(\bar{\alpha}^{j}\right) \leq 0}} R_{l_{s_{t}}, l_{s_{t}}}^{+}\left(g^{j}\left(\bar{\alpha}^{j}\right)\right)\left(\bar{p}-\bar{p}_{l_{s_{t}}}^{j}\right) \\
& =\sum_{l s_{t} \mid g_{l_{s_{t}}\left(\bar{\alpha}^{j}\right)>0}} R_{l s_{t}, l_{s_{t}}}^{+}\left(g^{j}\left(\bar{\alpha}^{j}\right)\right)\left(\bar{p}-\bar{p}_{l_{s_{t}}}^{j}\right)+\sum_{l s_{t} \mid g_{l_{s_{t}}\left(\bar{\alpha}^{j}\right) \leq 0}}\left(\bar{p}-\bar{p}_{l_{s_{t}}}^{j}\right) \\
& \leq \sum_{l_{s} \in \mathcal{L} \times \mathcal{N}}\left(\bar{p}_{l s_{t}}-\bar{p}_{l_{s_{t}}}^{j}\right)=0
\end{aligned}
$$

We have shown that

$$
\bar{p}^{\top} R^{+}\left(g^{j}\left(\bar{\alpha}^{j}\right)\right) g^{j}\left(\bar{\alpha}^{j}\right) \geq \bar{p}^{j^{\top}} R^{+}\left(g^{j}\left(\bar{\alpha}^{j}\right)\right) g^{j}\left(\bar{\alpha}^{j}\right), \quad j \in \mathcal{J}
$$

By (37), $\bar{p}^{j} \in \varphi^{j}\left(g^{j}\left(\bar{\alpha}^{j}\right) ; \rho\left(\bar{x},\left(g^{j}\left(\bar{\alpha}^{j}\right)\right){ }_{j \in \mathcal{J}}, \bar{p}\right)\right.$, so $\bar{p}^{j^{\top}} R^{+}\left(g^{j}\left(\bar{\alpha}^{j}\right)\right) g^{j}\left(\bar{\alpha}^{j}\right) \geq 0$ and, therefore,

$$
\bar{p}^{\top} R^{+}\left(g^{j}\left(\bar{\alpha}^{j}\right)\right) g^{j}\left(\bar{\alpha}^{j}\right) \geq 0 \quad \text { for } j \in \mathcal{J}
$$

By (43), dividend income is non-negative for every household, so the usual budget constraints apply. Summing over all budget constraints we get

$$
\bar{p}^{\top}\left(\sum_{h \in \mathcal{H}} \bar{x}^{h}-\sum_{h \in \mathcal{H}} e^{h}-\sum_{j \in \mathcal{J}} g^{j}\left(\bar{\alpha}^{j}\right)\right) \leq 0,
$$

which, combined with (36), yields

$$
\sum_{h \in \mathcal{H}} \bar{x}^{h}-\sum_{h \in \mathcal{H}} e^{h}-\sum_{j \in \mathcal{J}} g^{j}\left(\bar{\alpha}^{j}\right) \leq 0,
$$

Therefore, the fixed point defines a feasible allocation. It now follows that $\rho\left(\bar{x},\left(g^{j}\left(\bar{\alpha}^{j}\right)\right)_{j \in \mathcal{J}}\right)=\left(\bar{x},\left(g^{j}\left(\bar{\alpha}^{j}\right)\right)_{j \in \mathcal{J}}\right)$, so by (37) and (38),

$$
\begin{aligned}
& \bar{p}^{j} \in \varphi^{j}\left(g^{j}\left(\bar{\alpha}^{j}\right) ;\left(\bar{x},\left(g^{j^{\prime}}\left(\bar{\alpha}^{j^{\prime}}\right)\right)_{j^{\prime} \in \mathcal{J}}, \bar{p}\right), \quad j \in \mathcal{J},\right. \\
& \left.\bar{p}^{k} \in \tilde{\varphi}^{k}\left(\bar{y}^{k} ;\left(\bar{x},\left(g^{j}\left(\bar{\alpha}^{j}\right)\right)_{j \in \mathcal{J}}\right)\right), \bar{p}\right), \quad k \in \mathcal{K} .
\end{aligned}
$$

It also follows that $\bar{\alpha}^{j} \gg 0$ for all $j \in \mathcal{J}$, so by (39), $\bar{p}^{j}=\bar{p}$ for all $j \in \mathcal{J}$, so we have shown that

$$
\bar{p} \in \varphi^{j}\left(g^{j}\left(\bar{\alpha}^{j}\right) ;\left(\bar{x},\left(g^{j^{\prime}}\left(\bar{\alpha}^{j^{\prime}}\right)\right)_{j^{\prime} \in \mathcal{J}}, \bar{p}\right), \quad j \in \mathcal{J},\right.
$$

Equation (40) implies, for $l s_{t} \in \mathcal{L}^{k} \times \mathcal{N}$,

$$
\begin{array}{ll}
\bar{y}_{l s_{t}}^{k} \leq \bar{y}_{l s_{t}}^{k}+\bar{p} l_{s_{t}}-\bar{p}_{l s_{t}}^{k} & \text { if } \bar{y}_{l s_{t}}^{k}=\hat{y}_{l s_{t}}^{k}, \\
\bar{y}_{l s_{t}}^{k}=\bar{y}_{l s_{t}}^{k}+\bar{p} l_{s_{t}}-\bar{p}_{l s_{t}}^{k} & \text { if } 0<\bar{y}_{l s_{t}}^{k}<\hat{y}_{l s_{t}}^{k}, \\
\bar{y}_{l_{s_{t}}}^{k} \geq \bar{y}_{l s_{t}}^{k}+\bar{p} l_{s_{t}}-\bar{p}_{l_{s_{t}}}^{k} & \text { if } \bar{y}_{l s_{t}}^{k}=0
\end{array}
$$

whereas $\bar{y}_{l s_{t}}^{k}=0$ for $l s_{t} \in(\mathcal{L} \times \mathcal{N}) \backslash\left(\mathcal{L}^{k} \times \mathcal{N}\right)$. It follows that

$$
\left(\bar{p}-\bar{p}^{k}\right)^{\top} \bar{y}^{k} \geq 0, \quad \text { for all } k \in \mathcal{K},
$$




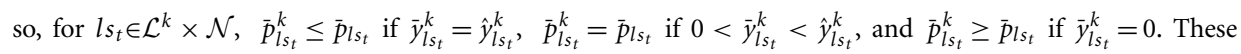
inequalities combined with (44) imply

$$
\bar{p} \in \varphi^{k}\left(\bar{y}^{k} ;\left(\bar{x},\left(g^{j}\left(\bar{\alpha}^{j}\right)\right){ }_{j \in \mathcal{J}}, \bar{p}\right), \quad k \in \mathcal{K} .\right.
$$

Suppose there is $l s_{t}$ such that $\bar{p} l s_{t}=0$. Let $l^{\prime} s_{t^{\prime}}^{\prime}$ be such that $\bar{p}_{l^{\prime} s_{t^{\prime}}^{\prime}}>0$. Let $h^{\prime}$ be a household whose supply of $l^{\prime} s_{t^{\prime}}^{\prime}$ is not under the control of a union. Our assumptions on primitives guarantee that there is $\hat{x}^{h^{\prime}} \in \gamma^{h^{\prime}}(\bar{p}, \bar{\alpha}, \bar{y} \mathcal{K}, \bar{x})$ with $\quad \bar{p}^{\top} R^{\circ}\left(\hat{z}^{h^{\prime}}\right) \hat{z}^{h^{\prime}}<0 \leq \sum_{j \in \mathcal{J}} \theta^{h^{\prime} j} \hat{v}^{j}+\theta^{h b} \hat{v}^{b}$, where $\quad \hat{v}^{j}=\bar{p}^{\top} R^{+}\left(g^{j}\left(\bar{\alpha}^{j}\right)\right) g^{j}\left(\bar{\alpha}^{j}\right), \quad j \in \mathcal{J}, \quad$ and $\hat{v}^{b}=\sum_{h \in \mathcal{H}} \bar{p}^{\top}\left(I-R^{\circ}\left(\bar{z}^{h}\right)\right) \bar{z}^{h}+\sum_{j \in \mathcal{J}} \bar{p}^{\top}\left(I-R^{+}\left(g^{j}\left(\bar{\alpha}^{j}\right)\right)\right) g^{j}\left(\bar{\alpha}^{j}\right)$. It follows that $\xi^{h^{\prime}}\left(\bar{p}, \bar{\alpha}, \bar{y}^{\mathcal{K}}, \bar{x}\right)$ is the set of $\succeq_{h^{\prime}}$-maximizers on $\gamma^{h^{\prime}}(\bar{p}, \bar{\alpha}, \bar{y} \mathcal{K}, \bar{x})$. Because preferences of $h^{\prime}$ are strictly monotonic with respect to $x_{l_{s}}^{h^{\prime}}$, it holds that $\bar{x}_{l_{s}}^{h^{\prime}}=c$, which contradicts that the fixed point defines a feasible allocation. Prices $\bar{p}$ are therefore strictly positive, all households can be shown to maximize their preferences, and

$$
\sum_{h \in \mathcal{H}} \bar{x}^{h}-\sum_{h \in \mathcal{H}} e^{h}-\sum_{j \in \mathcal{J}} g^{j}\left(\bar{\alpha}^{j}\right)=0 .
$$

Proof of Theorem 6: Going from the case of Theorem 5 with $\iota^{\top} \bar{p}=1$ to the case of Theorem 6 with $\iota^{\top} \bar{p}=\gamma$ for arbitrary $\gamma>0$ is a small step: just multiply all prices, vectors $\bar{q}^{j}, \bar{q}^{k}$ and matrices $H^{j-}(\cdot), H^{j+}(\cdot), \bar{H}^{j}(\cdot), H^{k-}(\cdot), H^{k+}(\cdot), \bar{H}^{k}(\cdot)$ by $\gamma$ and note that all the conditions in the definition of a Keynes-Negishi equilibrium remain satisfied. Indeed:

Ad (i): The budget sets are invariant with respect to $\gamma$; hence, $x^{h}$ is $\succeq_{h}$-maximal on the redefined feasible set.

Ad (ii): The profits are homogeneous of degree 1 in $\gamma$ because of Assumption A.8; hence, $y^{j}$ maximizes profits on $Y^{j}$ independent of the choice of $\gamma$.

Ad (iii): Assumption A.8 implies that the set of consumption bundles attainable for union members after redistribution is unaffected by $\gamma$; hence, $y^{k}$ is Pareto efficient for $\mathcal{H}^{k}$ independent of the choice of $\gamma$.

Ad (iv): Obvious.

\section{References}

Arnsperger, C., and D. de la Croix (1993), "Bargaining and equilibrium unemployment: Narrowing the gap between New Keynesian and disequilibrium theories," European Journal of Political Economy 9, 163-90.

Arrow, K. J. (1959), “Toward a theory of price adjustment,” M. Abramovitz, Allocation of Economic Resources, 41-51, Stanford: Stanford University Press.

Bénassy, J. P. (2002), The Macroeconomics of Imperfect Competition and Nonclearing Markets: A Dynamic General Equilibrium Approach, Cambridge: MIT Press.

Blair, D. H., and D. L. Crawford (1984), "Labor union objectives and collective bargaining," Quarterly Journal of Economics 99, 547-66.

Blanchard, O. J., and N. Kiyotaki (1987), "Monopolistic competition and the effects of aggregate demand," American Economic Review 77, 647-66.

Calmfors, L. (1982), "Employment policies, wage formation and trade union behavior in a small open economy," Scandinavian Journal of Economics 84, 345-73.

Citanna, A., H. Crès, J. H. Drèze, P. J. J. Herings, and A. Villanacci (2001), "Continua of underemployment equilibria reflecting coordination failures, also at Walrasian prices," Journal of Mathematical Economics 36, 169-200.

Clarke, F. H. (1983), Optimization and Nonsmooth Analysis, New York: John Wiley.

Clower, R. (1967), “A reconsideration of the microfoundations of monetary theory,” Western Economic Journal $6,1-8$. 
Dehez, P., and J. H. Drèze (1988), “Competitive equilibria with quantity-taking producers and increasing returns to scale," Journal of Mathematical Economics 17, 209-30.

Dehez P., J. H. Drèze, and T. Suzuki (2003), "Imperfect competition à la Negishi, also with fixed costs," Journal of Mathematical Economics 39, 219-37.

Dierker, E., R. Guesnerie, and W. Neuefeind (1985), "General equilibrium when some firms follow special pricing rules," Econometrica 53, 1369-94.

Drèze, J. H. (1974), “Investment under private ownership: optimality, equilibrium and stability,” J.H. Drèze, ed., Allocation under Uncertainty: Equilibrium and Optimality, 129-66, London: Macmillan.

Drèze, J. H. (1979), “Demand estimation, risk-aversion and sticky prices,” Economics Letters 4, 1-6.

Drèze, J. H. (1984), "Second-best analysis with markets in disequilibrium: Public sector pricing in a Keynesian regime,” European Economic Review 29, 263-301.

Drèze, J.H. (1991), "Stability of a Keynesian adjustment process," W. Barnett, B. Cornet, C. d'Aspremont, J. Jaskold Gabszevicz, and A. Mas-Colell, Equilibrium Theory and Applications, 197-231, Cambridge: Cambridge University Press.

Drèze, J.H. (1997), "Walras-Keynes equilibria, coordination and macroeconomics," European Economic Review 41, 1735-62.

Drèze, J. H. (1999), “On the dynamics of supply-constrained equilibria,” P.J.J. Herings, G. van der Laan, and A.J.J. Talman, The Theory of Markets, 7-26, Amsterdam: North-Holland.

Drèze, J. H. (2001), "On the macroeconomics of uncertainty and incomplete markets," Recherches Economiques de Louvain 67, 5-30. Reprinted as chapter 3 in J. H. Drèze, Advances in Macroeconomic Theory, International Economic Association Volume No. 133, 30-55, Basingstoke: Palgrave Macmillan.

Drèze, J. H., and C. Gollier (1993), "Risk sharing on the labour market and second-best wage rigidities," European Economic Review 37, 1457-82.

Drèze, J. H., and H. M. Polemarchakis (1999), “Money and monetary policy in general equilibrium,” A. P. Kirman, and L. A. Gérard-Varet, Economics Beyond the Millennium, 55-77, Oxford: Oxford University Press.

Drèze, J. H., and H. M. Polemarchakis (2001a), "Intertemporal general equilibrium and monetary theory," A. Leijonhufvud, Monetary Theory as a Basis for Monetary Policy, 33-71, London: Macmillan.

Drèze, J. H., and H. M. Polemarchakis (2001b), "Monetary equilibria," G. Debreu, W. Neuefeind, and W. Trockel, Economics Essays: A Festschrift for Werner Hildenbrand, 83-108, Heidelberg: Springer-Verlag.

Dunlop, J. T. (1944), Wage Determination under Trade Unions, New York: MacMillan.

Farber, H. S. (1986), “The analysis of union behavior,” O.C. Ashenfelter and R. Layard, Handbook of Labor Economics, Volume II, 1039-1089, Amsterdam: North-Holland.

Greenberg, J. (1977), “Quasi-equilibrium in abstract economies without ordered preferences,” Journal of Mathematical Economics 4, 163-65.

Hart, O. D. (1982), “A model of imperfect competition with Keynesian features," Quarterly Journal of Economics 97, 109-38.

Herings, P. J. J. (1996), Static and Dynamic Aspects of General Disequilibrium Theory, Theory and Decision Library, Series C: Game Theory, Mathematical Programming and Operations Research, Norwell: Kluwer Academic Publishers.

Herings, P. J. J., and H. M. Polemarchakis (2005), "Pareto improving price regulation when the asset market is incomplete," Economic Theory 25, 135-54.

Hicks, J. R. (1974), The Crisis in Keynesian Economics, Oxford: Blackwell.

Hildenbrand, W. (1974), Core and Equilibria of a Large Economy, Princeton: Princeton University Press.

Licandro, O. (1995), “A non-Walrasian general equilibrium model with monopolistic competition and wage bargaining," Annales d'Economie et de Statistique 37/38, 237-53.

Magill, M., and M. Quinzii (1996), Theory of Incomplete Markets, Cambridge: MIT Press.

Negishi, T. (1961), “Monopolistic competition and general equilibrium,” Review of Economic Studies 28, $196-201$.

Negishi, T. (1972), General Equilibrium and International Trade, Amsterdam: North-Holland.

Negishi, T. (1974), “Involuntary unemployment and market imperfection,” Economic Studies Quarterly 25, 32-41. Negishi, T. (1976), “The excess of productive public expenditures,” Zeitschrift für Nationalökonomie 36, 85-94. 
Negishi, T. (1979), Microeconomic Foundations of Keynesian Macroeconomics, Studies in Mathematical and Managerial Economics, Amsterdam: North-Holland.

Oswald, A. T. (1979), "Wage determination in an economy with many trade unions," Oxford Economic Papers 31, 369-85.

Oswald, A. J. (1985), “The economic theory of trade unions: an introductory survey," Scandinavian Journal of Economics 87/2, 160-93.

Rockafellar, R. T. (1970), Convex Analysis, Princeton: Princeton University Press.

Snower, D. (1983), "Imperfect competition, unemployment and crowding-out," Oxford Economic Papers, Supplement 35, 245-70.

Stiglitz, J. E. (1984), “Price rigidities and market structure,” American Economic Review 74, 350-55.

Sweezy, P. M. (1939), "Demand under conditions of oligopoly,” Journal of Political Economy 47, 568-73.

Woodford, M. (1991), "Self-fulfilling expectations and fluctuations in aggregate demand,” N. G. Mankiw and D. Romer, New Keynesian Economics, Volume II, 77-102, Cambridge: MIT Press.

Woodford, M. (2003), Interest \& Prices, Foundations of a Theory of Monetary Policy, Princeton: Princeton University Press. 